\title{
Structure determination, biosynthetic origin, and total synthesis of akazaoxime, an enteromycin-class metabolite from marine-derived actinomycete of the genus Micromonospora
}

Yasuhiro Igarashi*, Yoe Matsuyuki, Masayuki Yamada, Nodoka Fujihara, Enjuro Harunari, Naoya Oku, Md. Rokon Ul Karim, Taehui Yang, Katsuhisa Yamada, Chiaki Imada, Keisuke Fukaya, and Daisuke Urabe*

Biotechnology Research Center and Department of Biotechnology, Toyama Prefectural University, 5180 Kurokawa, Imizu, Toyama 939-0398, Japan

Graduate School of Marine Science and Technology, Tokyo University of Marine Science and Technology, 4-5-7, Konan, Minato-ku, Tokyo 108-8477, Japan

DHC Corporation, 2-7-1 Minami-Azabu, Minato-ku, Tokyo 106-8571, Japan

Supporting Information

48 pages

\section{Table of Contents}

page

Figure S1. UV spectrum of akazaoxime (1) S3

$\begin{array}{ll}\text { Figure S2. HRMS spectrum of } 1 & \text { S3 }\end{array}$

Figure S3. IR spectrum of $1 \quad$ S4

Figure S4. ${ }^{1} \mathrm{H}$ NMR spectrum of $\mathbf{1}\left(500 \mathrm{MHz}, \mathrm{CD}_{3} \mathrm{OD}\right) \quad$ S5

Figure S5. ${ }^{13} \mathrm{C}\left\{{ }^{1} \mathrm{H}\right\}$ NMR spectrum of $\mathbf{1}\left(125 \mathrm{MHz}, \mathrm{CD}_{3} \mathrm{OD}\right) \quad$ S6

Figure S6. COSY spectrum of $\mathbf{1}\left(500 \mathrm{MHz}, \mathrm{CD}_{3} \mathrm{OD}\right) \quad \mathrm{S} 7$

Figure S7. HSQC spectrum of $\mathbf{1}\left(500 \mathrm{MHz}, \mathrm{CD}_{3} \mathrm{OD}\right) \quad \mathrm{S} 8$

Figure S8. $\mathrm{HMBC}$ spectrum of $\mathbf{1}\left(500 \mathrm{MHz}, \mathrm{CD}_{3} \mathrm{OD}\right) \quad \mathrm{S} 9$

Figure S9. NOESY spectrum of $1\left(500 \mathrm{MHz}, \mathrm{CD}_{3} \mathrm{OD}\right) \quad \mathrm{S} 10$

Figure S10. ${ }^{1} \mathrm{H}$ NMR spectrum of $1\left(500 \mathrm{MHz}\right.$, acetone- $\left.d_{6}\right) \quad \mathrm{S} 11$

Figure S11. ${ }^{13} \mathrm{C}\left\{{ }^{1} \mathrm{H}\right\}$ NMR spectrum of $\mathbf{1}\left(125 \mathrm{MHz}\right.$, acetone- $\left.d_{6}\right) \quad$ S12

Figure S12. COSY spectrum of $1\left(500 \mathrm{MHz}\right.$, acetone- $\left.d_{6}\right) \quad$ S13

Figure S13. HSQC spectrum of $1\left(500 \mathrm{MHz}\right.$, acetone- $\left.d_{6}\right) \quad \mathrm{S} 14$

Figure S14. HMBC spectrum of $1\left(500 \mathrm{MHz}\right.$, acetone- $\left.d_{6}\right) \quad \mathrm{S} 15$

Figure S15. HRMS spectrum of A-76356 (2) S16

Figure S16. ${ }^{1} \mathrm{H}$ NMR spectrum of A-76356 (2) $\left(500 \mathrm{MHz}, \mathrm{CDCl}_{3}\right) \quad \mathrm{S} 17$

Figure S17. ${ }^{13} \mathrm{C}\left\{{ }^{1} \mathrm{H}\right\}$ NMR spectrum of $2\left(125 \mathrm{MHz}, \mathrm{CDCl}_{3}\right) \quad \mathrm{S} 18$

Figure S18. COSY spectrum of $2\left(500 \mathrm{MHz}, \mathrm{CDCl}_{3}\right) \quad \mathrm{S} 19$

Figure S19. HSQC spectrum of $2\left(500 \mathrm{MHz}, \mathrm{CDCl}_{3}\right) \quad$ S20

Figure S20. HMBC spectrum of $2\left(500 \mathrm{MHz}, \mathrm{CDCl}_{3}\right) \quad$ S21

Figure S21. NOESY spectrum of $2\left(500 \mathrm{MHz}, \mathrm{CDCl}_{3}\right) \quad$ S22

Figure S22. ${ }^{1} \mathrm{H}$ NMR spectrum of $\mathbf{3 a}\left(500 \mathrm{MHz}, \mathrm{CD}_{3} \mathrm{OD}\right) \quad$ S23

Figure S23. COSY spectrum of $\mathbf{3 a}\left(500 \mathrm{MHz}, \mathrm{CD}_{3} \mathrm{OD}\right) \quad$ S24

Figure S24. ${ }^{1} \mathrm{H}$ NMR spectrum of $\mathbf{3 b}\left(500 \mathrm{MHz}, \mathrm{CD}_{3} \mathrm{OD}\right) \quad$ S25

Figure S25. COSY spectrum of $\mathbf{3 b}\left(500 \mathrm{MHz}, \mathrm{CD}_{3} \mathrm{OD}\right) \quad \mathrm{S} 26$

Figure S26. ${ }^{13} \mathrm{C}\left\{{ }^{1} \mathrm{H}\right\}$ NMR spectra of 1 labelled with DL- $\left[2-{ }^{13} \mathrm{C}\right]$ leucine, $\left[1-{ }^{13} \mathrm{C}\right]$ propionate, $\left[1-{ }^{13} \mathrm{C}\right]$ acetate, and $\left[1-{ }^{13} \mathrm{C}\right]$ glycine $\left(125 \mathrm{MHz}, \mathrm{CDCl}_{3}\right) \quad$ S27

Figure S27. ${ }^{13} \mathrm{C}$ NMR spectra of 2 labelled with DL- $\left[2-{ }^{13} \mathrm{C}\right]$ leucine, $\left[1-{ }^{13} \mathrm{C}\right]$ propionate, $\left[1-{ }^{13} \mathrm{C}\right]$ acetate, and $\left[1-{ }^{13} \mathrm{C}\right]$ glycine $\left(125 \mathrm{MHz}, \mathrm{CDCl}_{3}\right) \quad$ S28

Figure S28. ${ }^{1} \mathrm{H}$ NMR spectrum of $9\left(500 \mathrm{MHz}, \mathrm{C}_{6} \mathrm{D}_{6}, 336 \mathrm{~K}\right) \quad$ S29 
Figure S29. ${ }^{13} \mathrm{C}\left\{{ }^{1} \mathrm{H}\right\}$ NMR spectrum of $9\left(125 \mathrm{MHz}, \mathrm{C}_{6} \mathrm{D}_{6}, 336 \mathrm{~K}\right) \quad \mathrm{S} 30$

Figure S30. ${ }^{1} \mathrm{H}$ NMR spectrum of $\mathbf{1 1}\left(400 \mathrm{MHz}, \mathrm{CDCl}_{3}\right) \quad \mathrm{S} 31$

Figure S31. ${ }^{13} \mathrm{C}\left\{{ }^{1} \mathrm{H}\right\}$ NMR spectrum of $\mathbf{1 1}\left(100 \mathrm{MHz}, \mathrm{CDCl}_{3}\right) \quad$ S32

Figure S32. ${ }^{1} \mathrm{H}$ NMR spectrum of $\mathbf{1 4}\left(500 \mathrm{MHz}, \mathrm{C}_{6} \mathrm{D}_{6}, 333 \mathrm{~K}\right) \quad$ S33

Figure S33. ${ }^{13} \mathrm{C}\left\{{ }^{1} \mathrm{H}\right\}$ NMR spectrum of $\mathbf{1 4}\left(125 \mathrm{MHz}, \mathrm{C}_{6} \mathrm{D}_{6}, 333 \mathrm{~K}\right) \quad \mathrm{S} 34$

Figure S34. ${ }^{1} \mathrm{H}$ NMR spectrum of $\mathbf{1 5}\left(500 \mathrm{MHz}, \mathrm{CD}_{3} \mathrm{OD}\right) \quad \mathrm{S} 35$

Figure S35. ${ }^{13} \mathrm{C}\left\{{ }^{1} \mathrm{H}\right\}$ NMR spectrum of $\mathbf{1 5}\left(125 \mathrm{MHz}, \mathrm{CD}_{3} \mathrm{OD}\right) \quad \mathrm{S} 36$

Figure S36. ${ }^{1} \mathrm{H}$ NMR spectrum of synthetic $1\left(500 \mathrm{MHz}, \mathrm{CD}_{3} \mathrm{OD}\right) \quad \mathrm{S} 37$

Figure S37. ${ }^{13} \mathrm{C}\left\{{ }^{1} \mathrm{H}\right\}$ NMR spectrum of synthetic $1\left(125 \mathrm{MHz}, \mathrm{CD}_{3} \mathrm{OD}\right) \quad \mathrm{S} 38$

Figure S38. ${ }^{1} \mathrm{H}$ NMR spectrum of $\mathbf{1 7}\left(500 \mathrm{MHz}, \mathrm{CD}_{3} \mathrm{OD}\right) \quad \mathrm{S} 39$

Figure S39. ${ }^{13} \mathrm{C}\left\{{ }^{1} \mathrm{H}\right\}$ NMR spectrum of $17\left(125 \mathrm{MHz}, \mathrm{CD}_{3} \mathrm{OD}\right) \quad \mathrm{S} 40$

Figure S40. ${ }^{1} \mathrm{H}$ NMR spectrum of $\mathbf{1 8}\left(400 \mathrm{MHz}, \mathrm{CD}_{3} \mathrm{OD}\right) \quad \mathrm{S} 41$

Figure S41. ${ }^{13} \mathrm{C}\left\{{ }^{1} \mathrm{H}\right\}$ NMR spectrum of $18\left(100 \mathrm{MHz}, \mathrm{CD}_{3} \mathrm{OD}\right) \quad \mathrm{S} 42$

Figure S42. Comparison of ${ }^{1} \mathrm{H}$ NMR spectra of natural and synthetic $1\left(500 \mathrm{MHz}, \mathrm{CD}_{3} \mathrm{OD}\right) \quad \mathrm{S} 43$

Figure S43. Comparison of ${ }^{13} \mathrm{C}\left\{{ }^{1} \mathrm{H}\right\}$ NMR spectra of natural and synthetic 1

$\left(125 \mathrm{MHz}, \mathrm{CD}_{3} \mathrm{OD}\right)$

S44

Table S1. Structure, energy and Cartesian coordinate of 2a $\quad$ S45

Table S2. Structure, energy and Cartesian coordinate of $\mathbf{2 b} \quad$ S46

Table S3. X-ray crystallographic structure of $\mathbf{1 1}$ with thermal ellipsoids $\quad$ S47 
Figure S1. UV spectrum of akazaoxime (1)

(measured in $\mathrm{MeOH}$ )

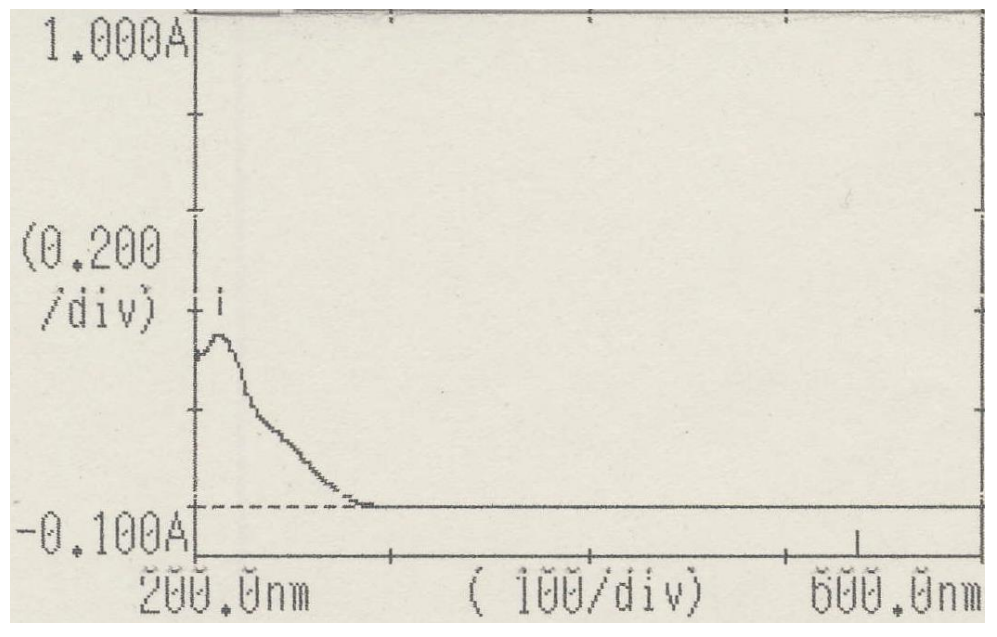

Figure S2. HRMS spectrum of $\mathbf{1}$

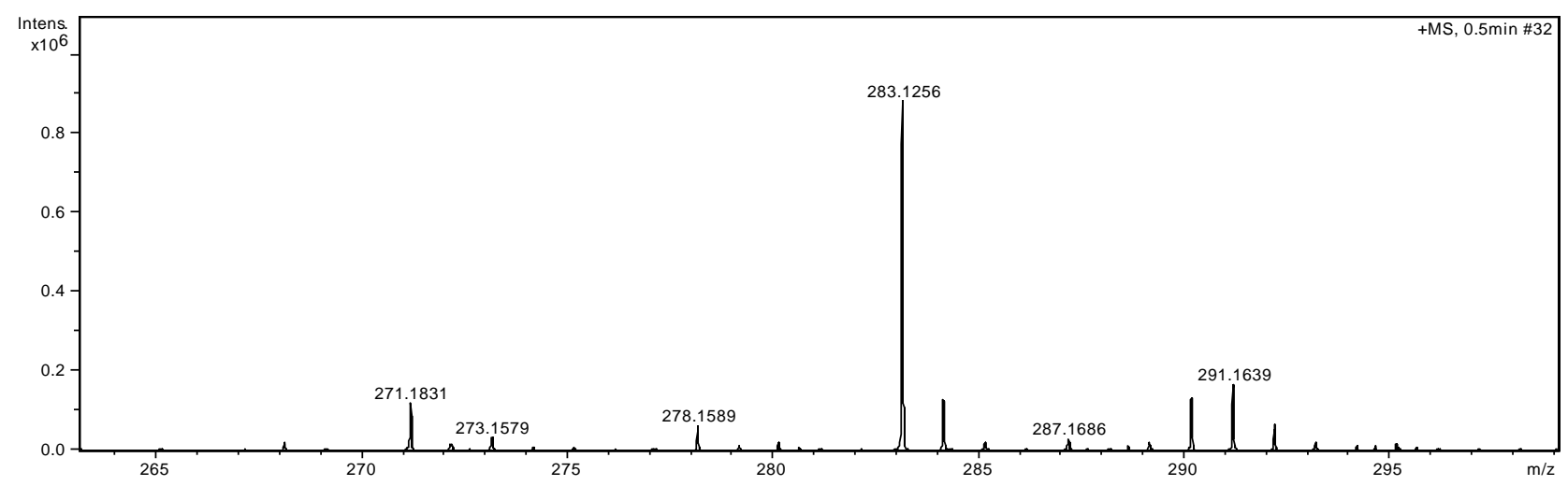


Figure S3. IR spectrum of 1

(ATR)

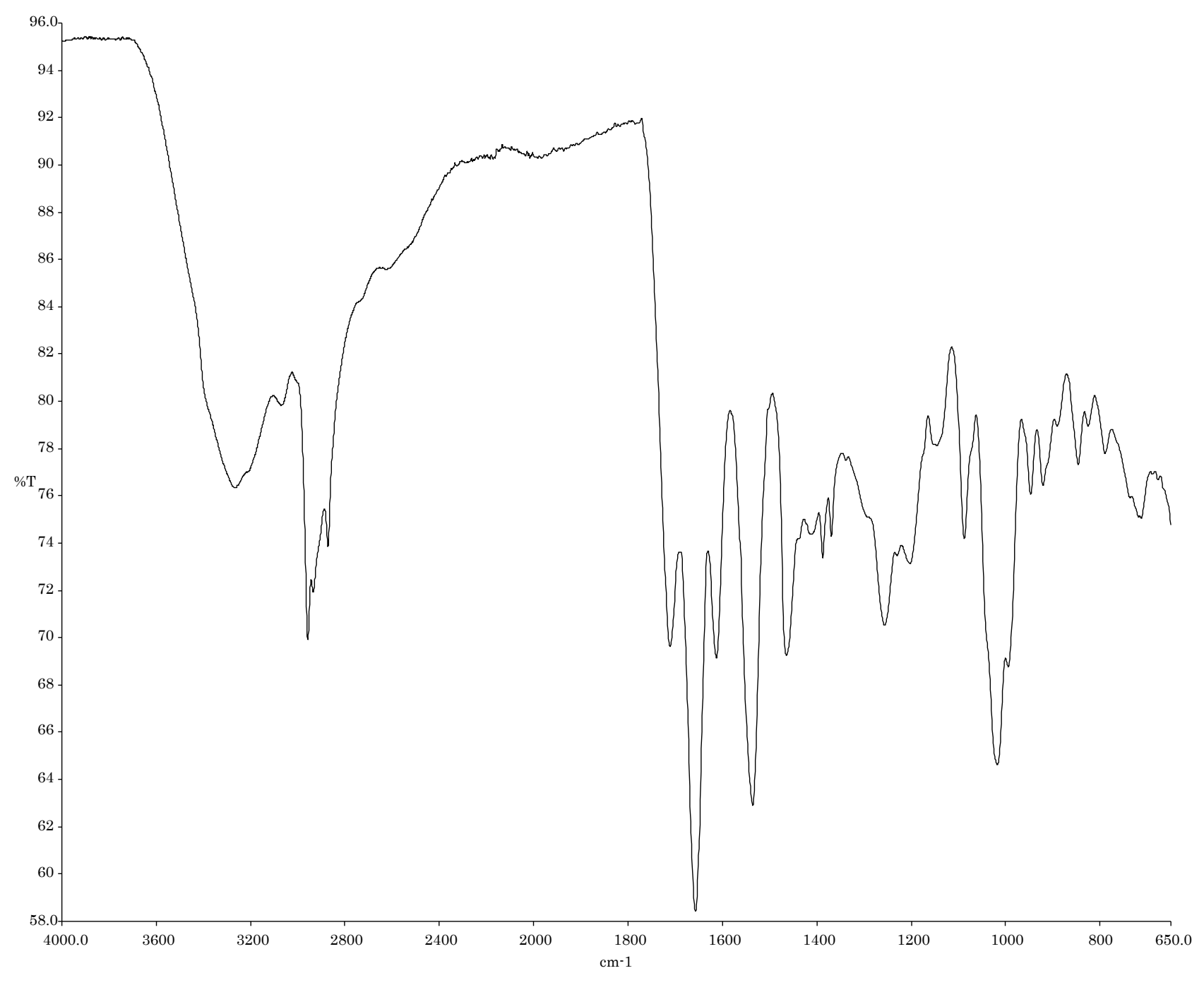


Figure S4. ${ }^{1} \mathrm{H}$ NMR spectrum of $\mathbf{1}\left(500 \mathrm{MHz}, \mathrm{CD}_{3} \mathrm{OD}\right)$
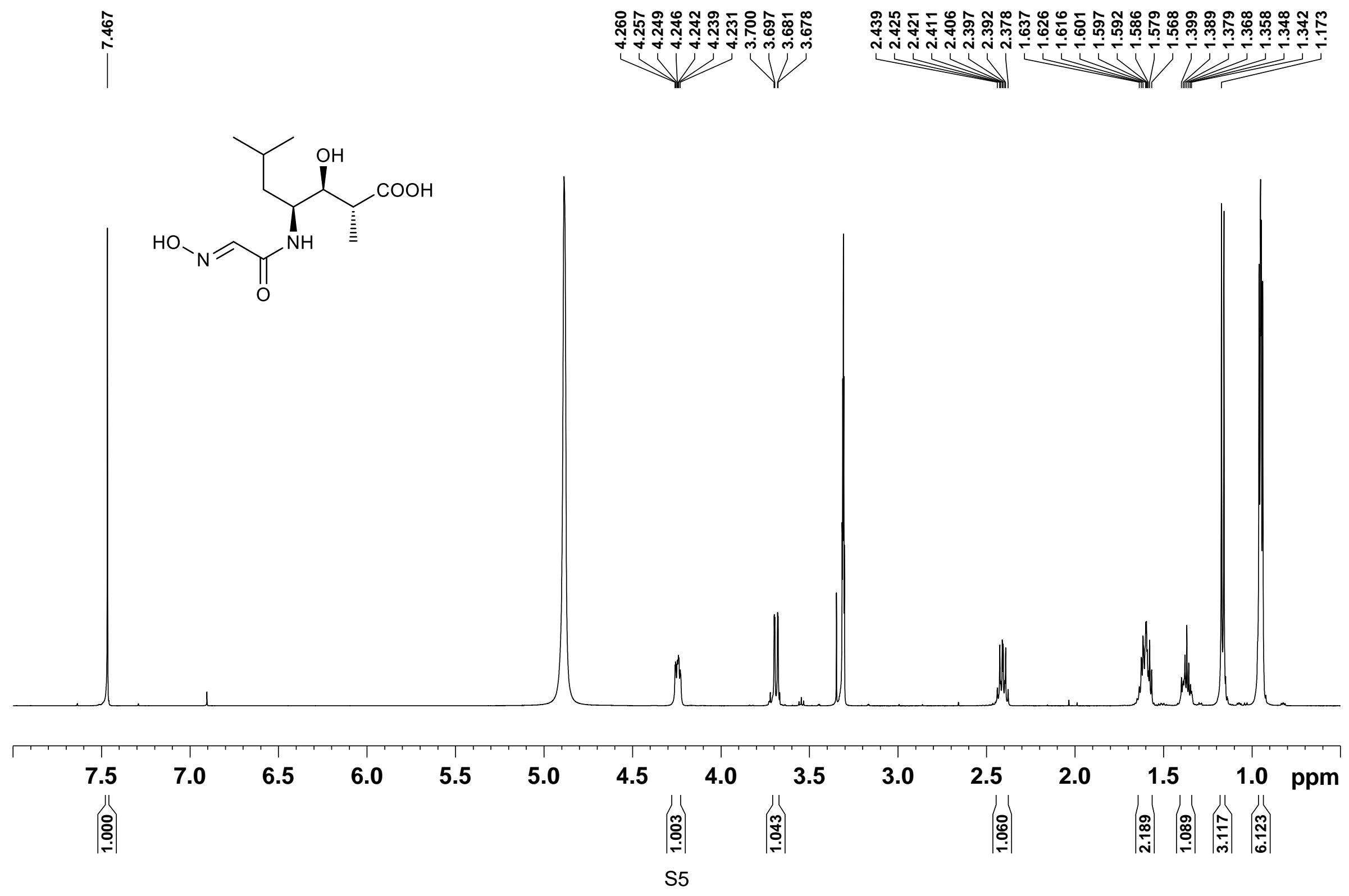
Figure S5. ${ }^{13} \mathrm{C}\left\{{ }^{1} \mathrm{H}\right\}$ NMR spectrum of $\mathbf{1}\left(125 \mathrm{MHz}, \mathrm{CD}_{3} \mathrm{OD}\right)$
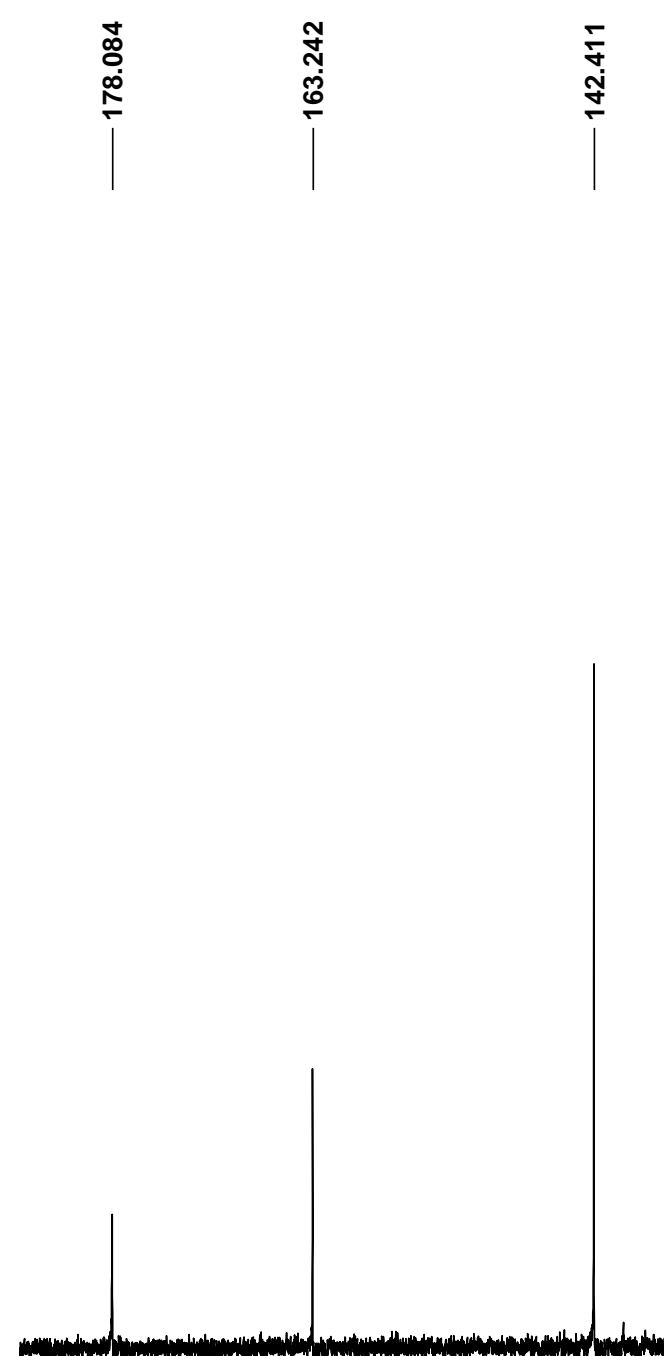

80


Figure S6. COSY spectrum of $1\left(500 \mathrm{MHz}, \mathrm{CD}_{3} \mathrm{OD}\right)$

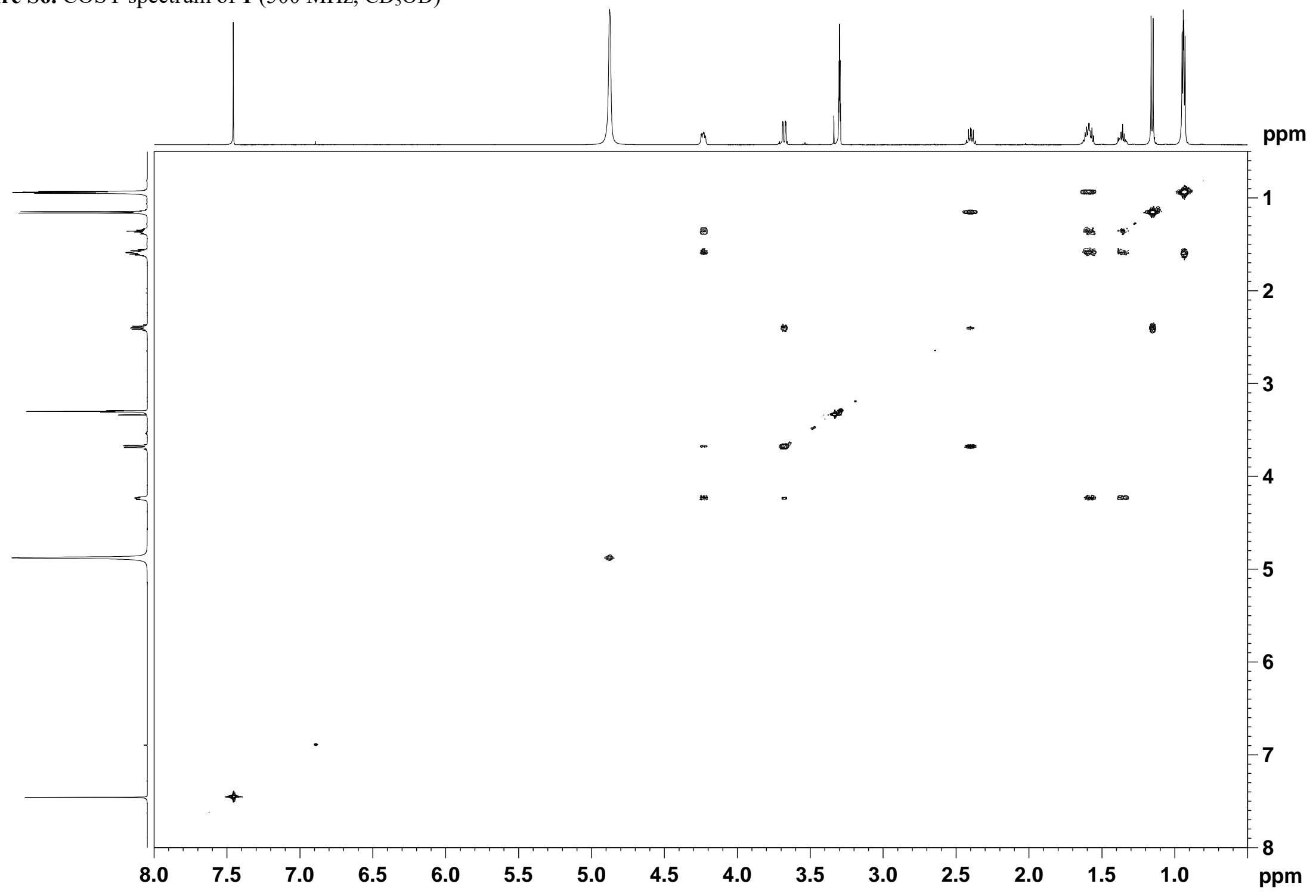


Figure S7. HSQC spectrum of $1\left(500 \mathrm{MHz}, \mathrm{CD}_{3} \mathrm{OD}\right)$

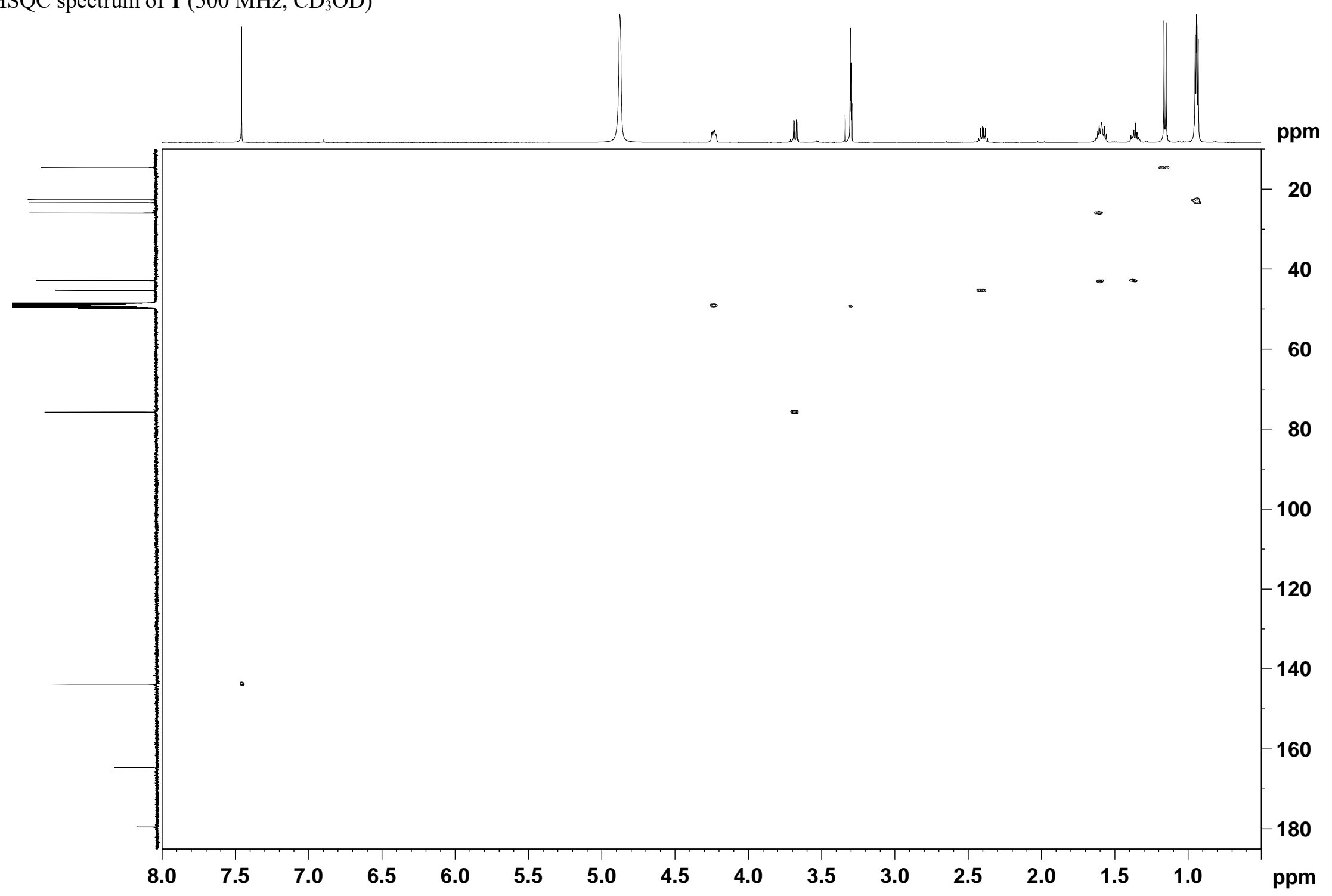


Figure S8. $\mathrm{HMBC}$ spectrum of $\mathbf{1}\left(500 \mathrm{MHz}, \mathrm{CD}_{3} \mathrm{OD}\right)$

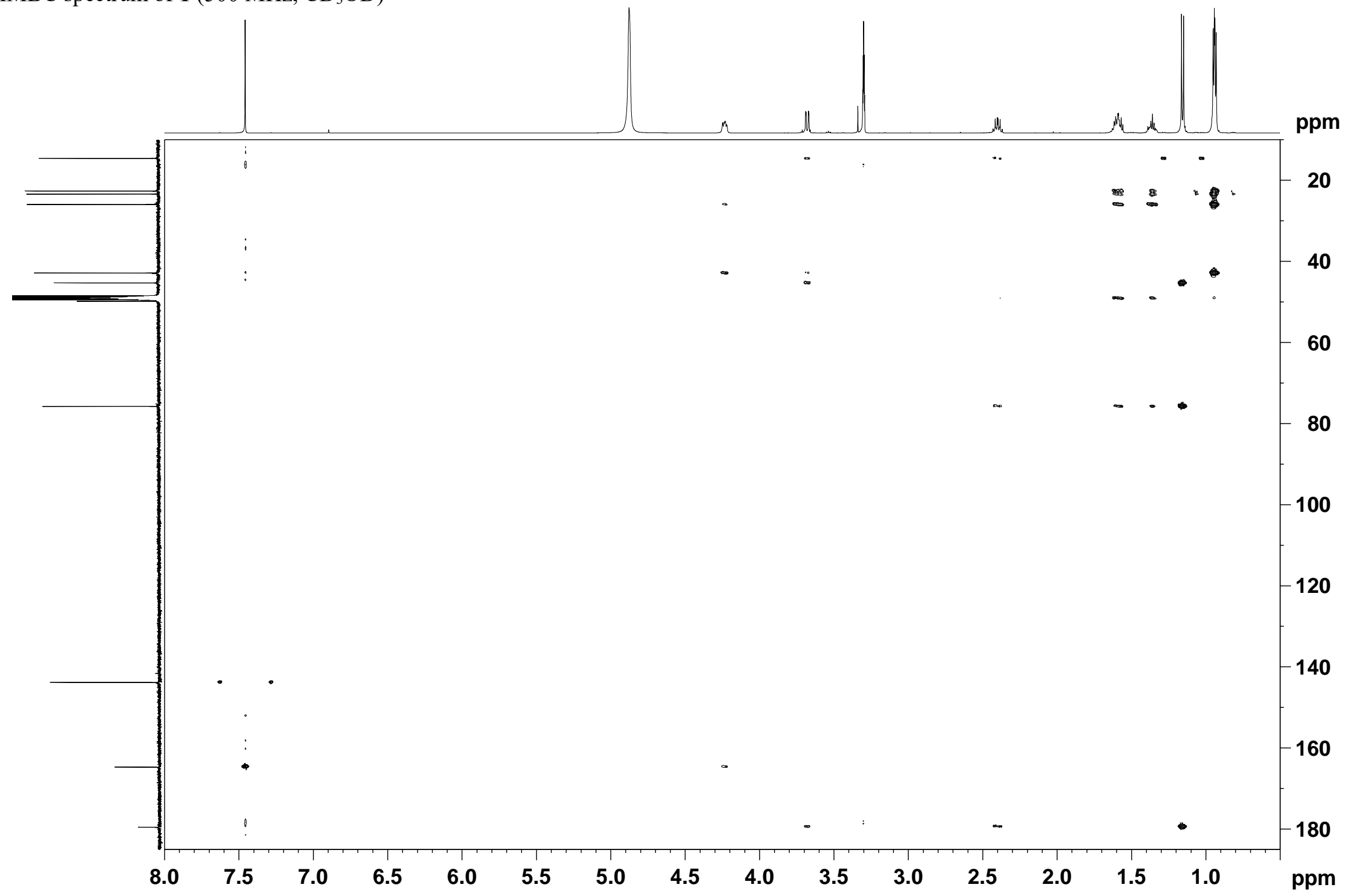


Figure S9. NOESY spectrum of $1\left(500 \mathrm{MHz}, \mathrm{CD}_{3} \mathrm{OD}\right)$

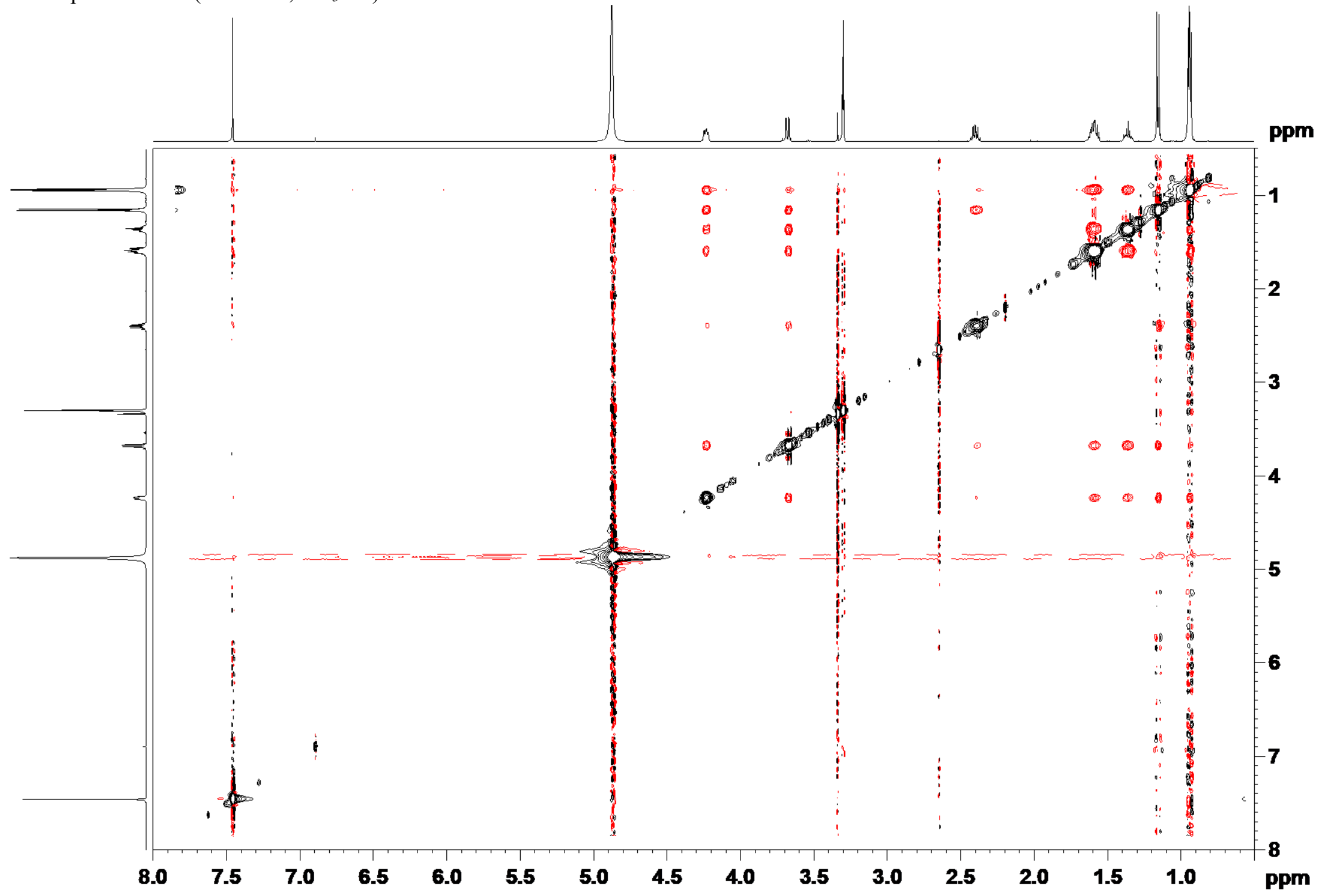

S10 
Figure S10. ${ }^{1} \mathrm{H}$ NMR spectrum of $1\left(500 \mathrm{MHz}\right.$, acetone- $d_{6}$ )

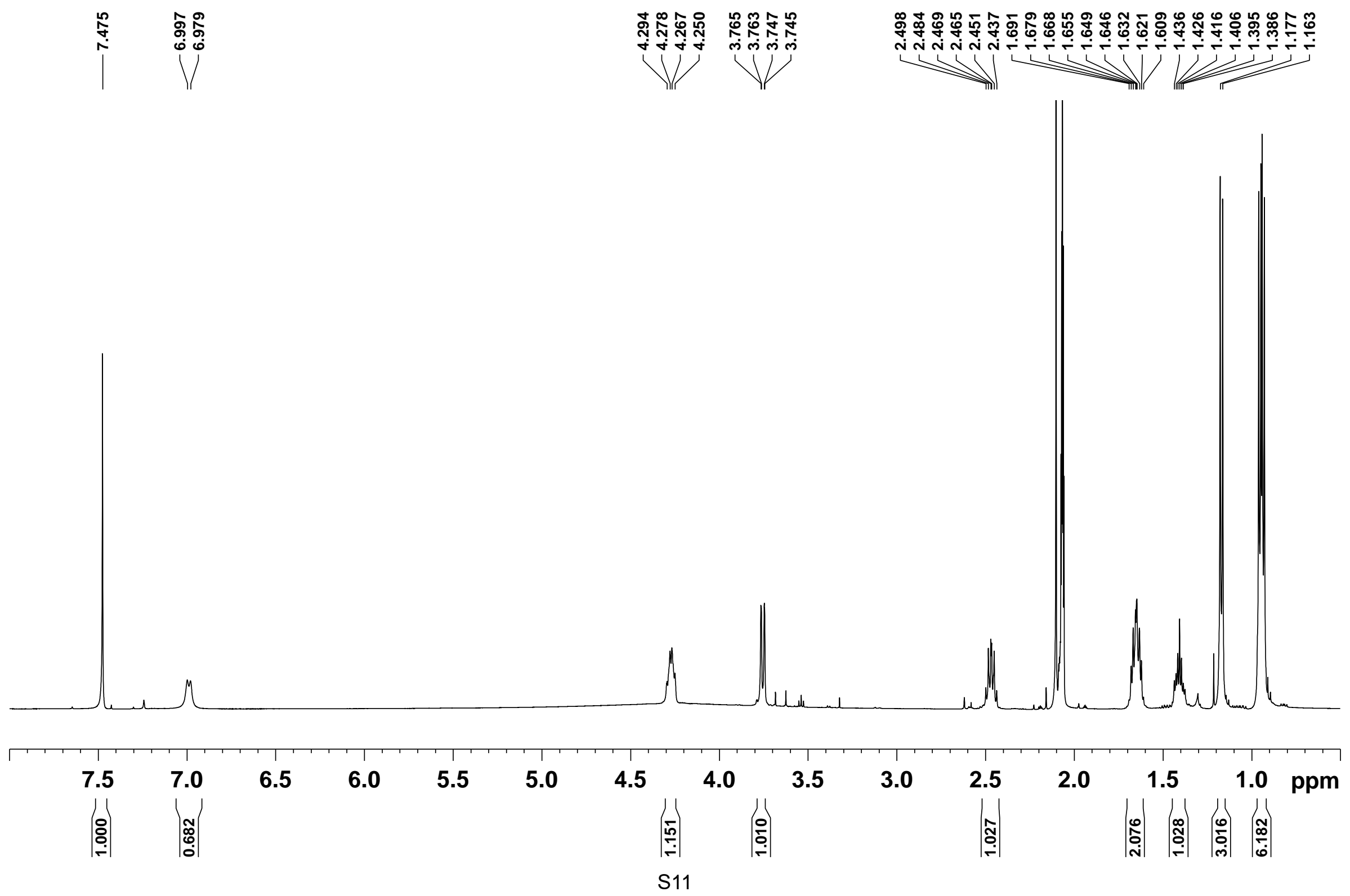


Figure S11. ${ }^{13} \mathrm{C}\left\{{ }^{1} \mathrm{H}\right\}$ NMR spectrum of $\mathbf{1}\left(125 \mathrm{MHz}\right.$, acetone- $\left.d_{6}\right)$

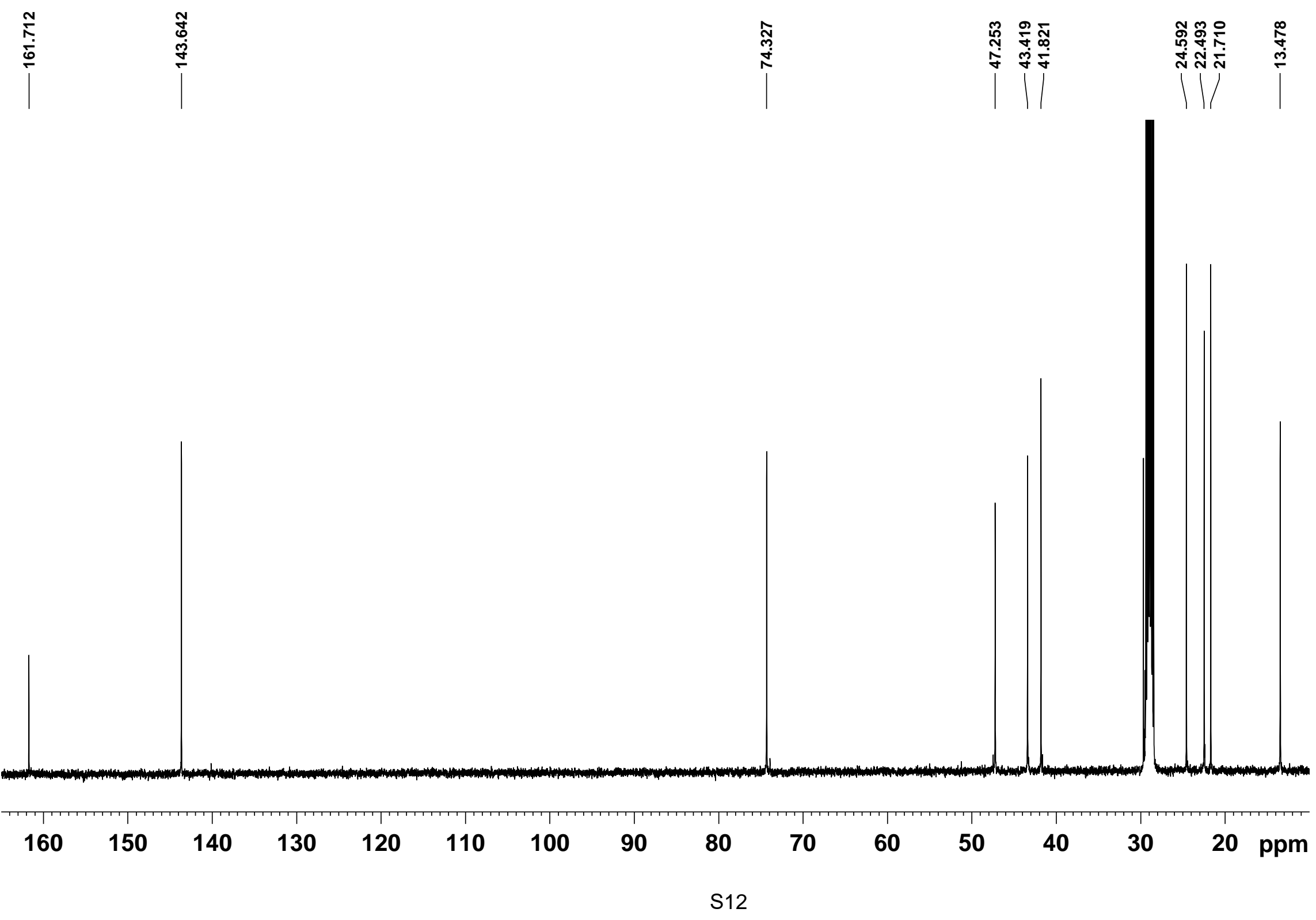


Figure S12. COSY spectrum of $1\left(500 \mathrm{MHz}\right.$, acetone- $\left.d_{6}\right)$

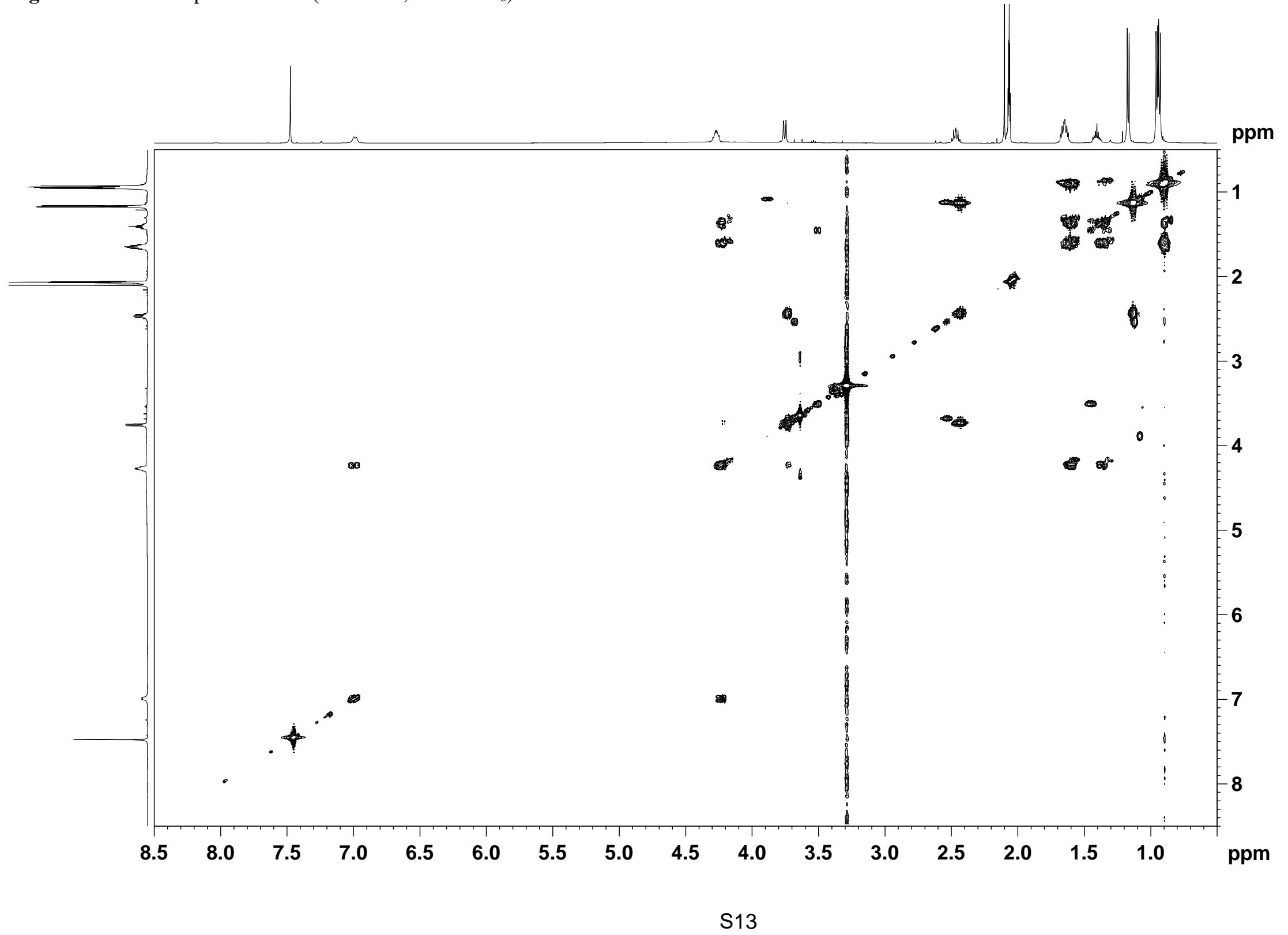


Figure S13. HSQC spectrum of $1\left(500 \mathrm{MHz}\right.$, acetone- $\left.d_{6}\right)$

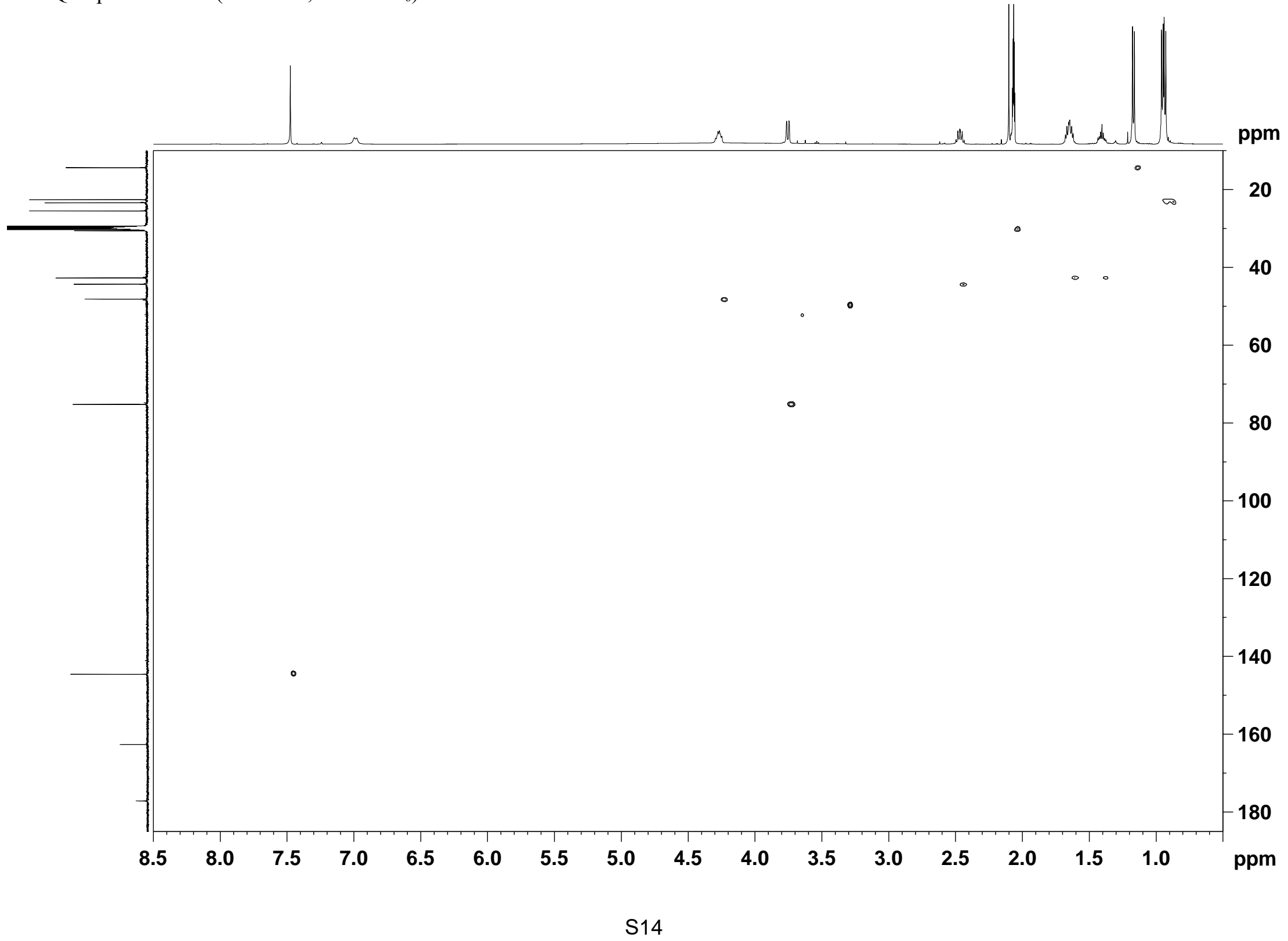


Figure S14. HMBC spectrum of 1 ( $500 \mathrm{MHz}$, acetone- $d_{6}$ )

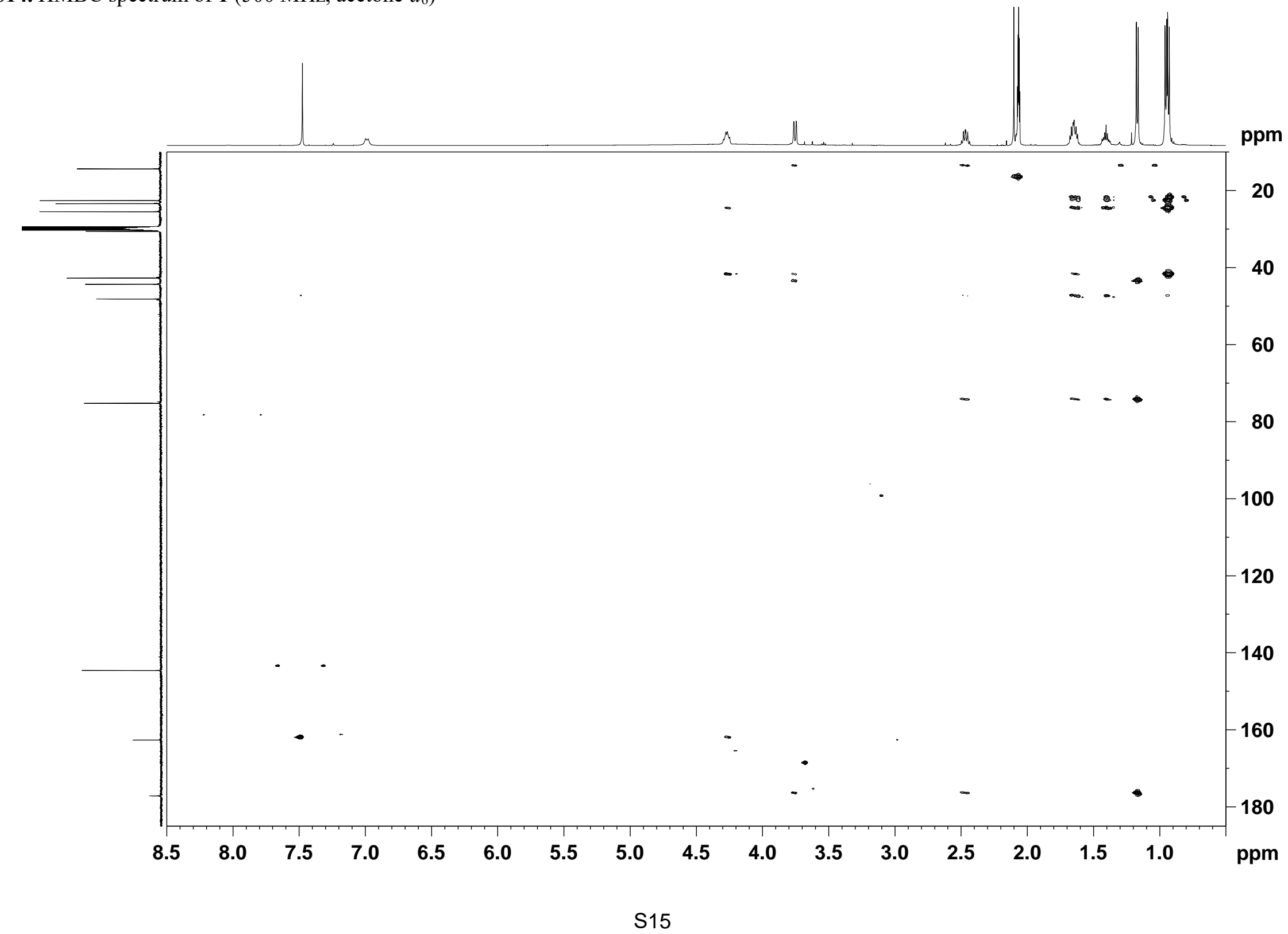


Figure S15. HRMS spectrum of A-76356 (2)

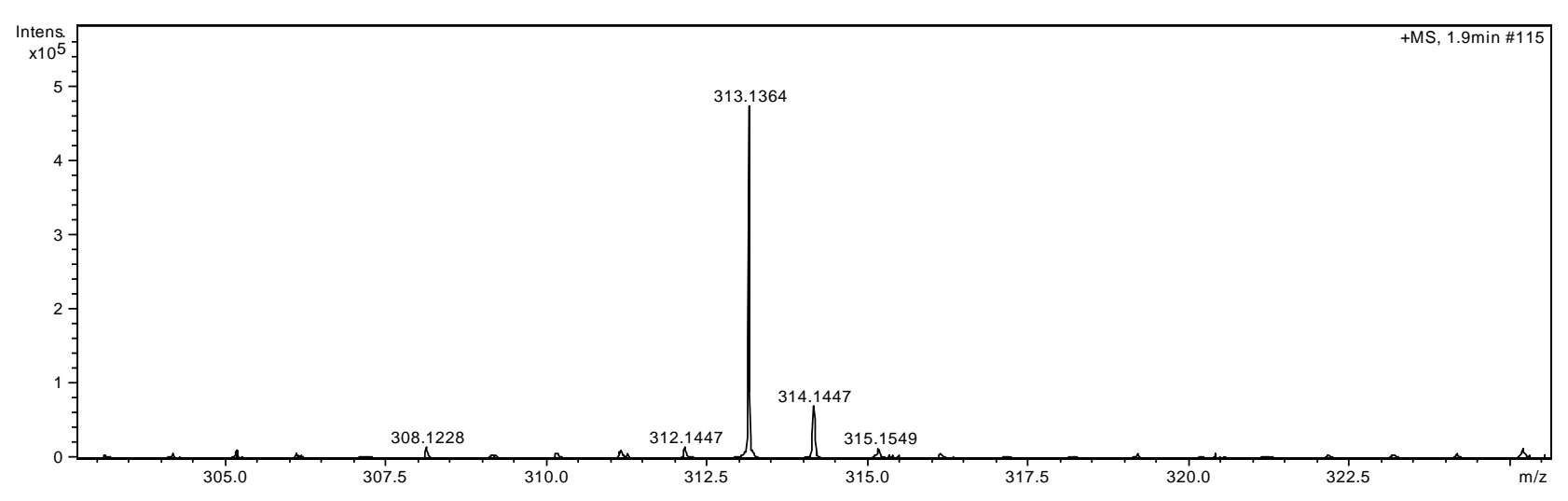


Figure S16. ${ }^{1} \mathrm{H}$ NMR spectrum of A-76356 (2) (500 MHz, $\left.\mathrm{CDCl}_{3}\right)$
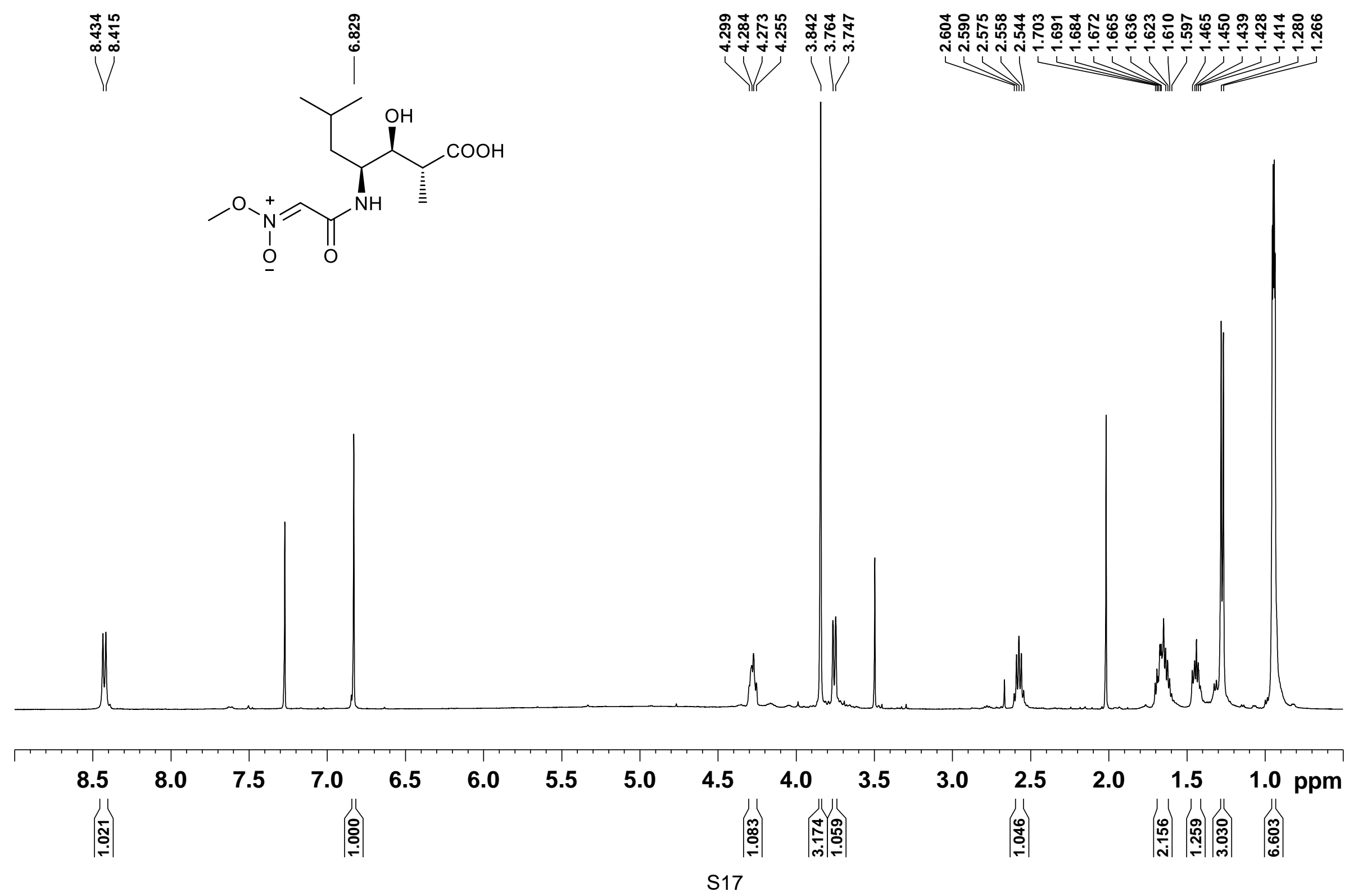
Figure S17. ${ }^{13} \mathrm{C}\left\{{ }^{1} \mathrm{H}\right\}$ NMR spectrum of $2\left(125 \mathrm{MHz}, \mathrm{CDCl}_{3}\right)$
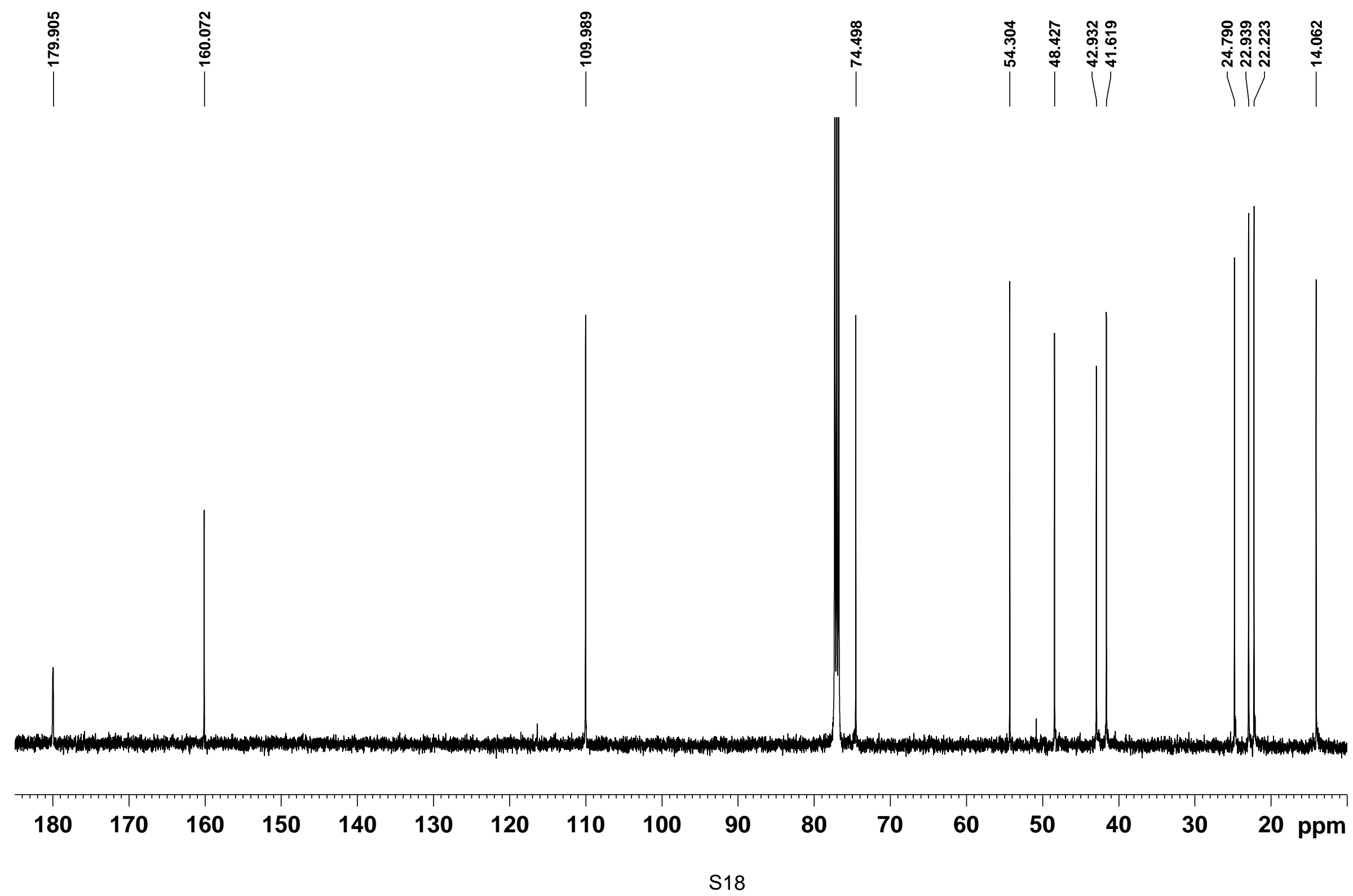
Figure S18. COSY spectrum of $2\left(500 \mathrm{MHz}, \mathrm{CDCl}_{3}\right)$

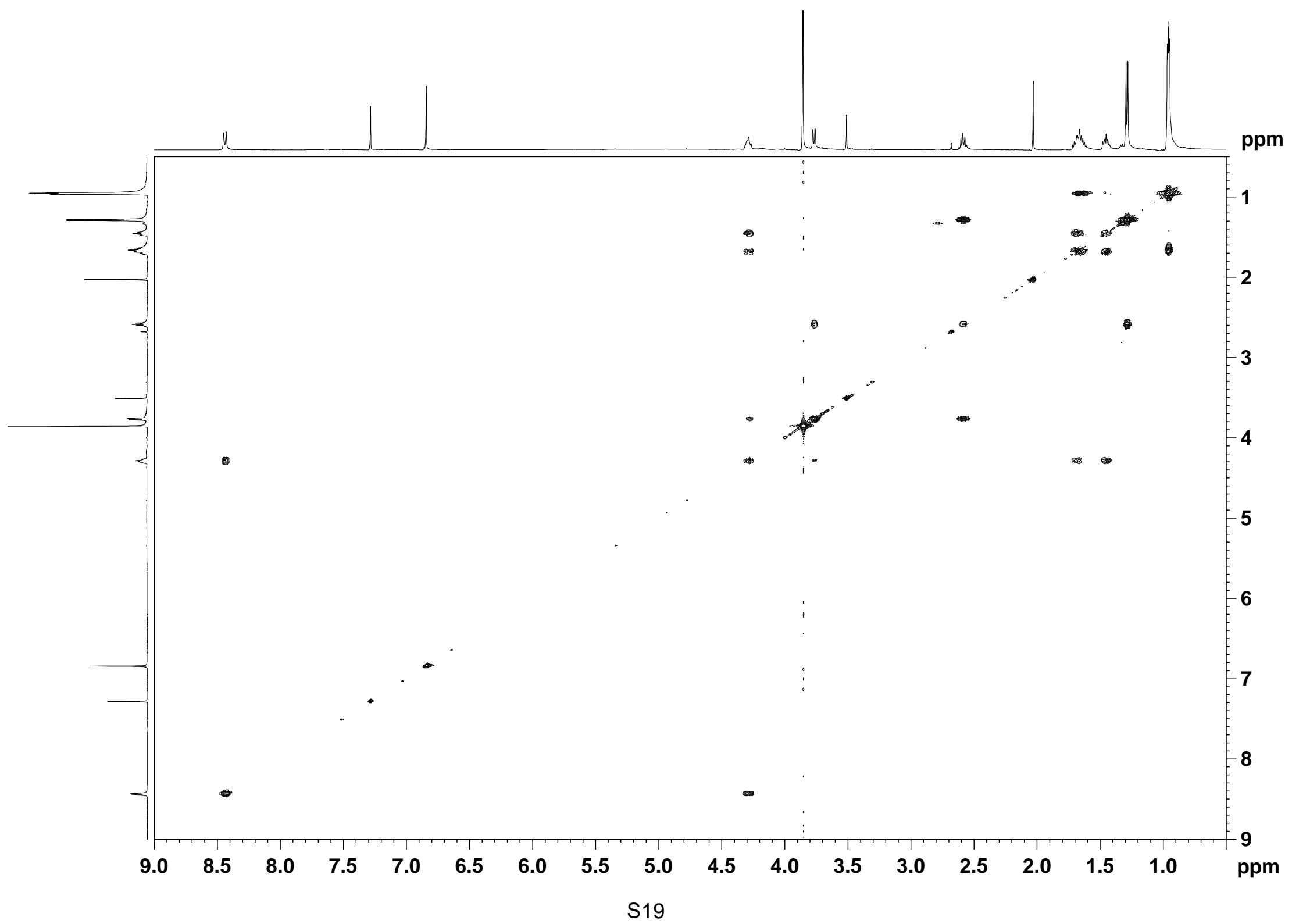


Figure S19. HSQC spectrum of $2\left(500 \mathrm{MHz}, \mathrm{CDCl}_{3}\right)$

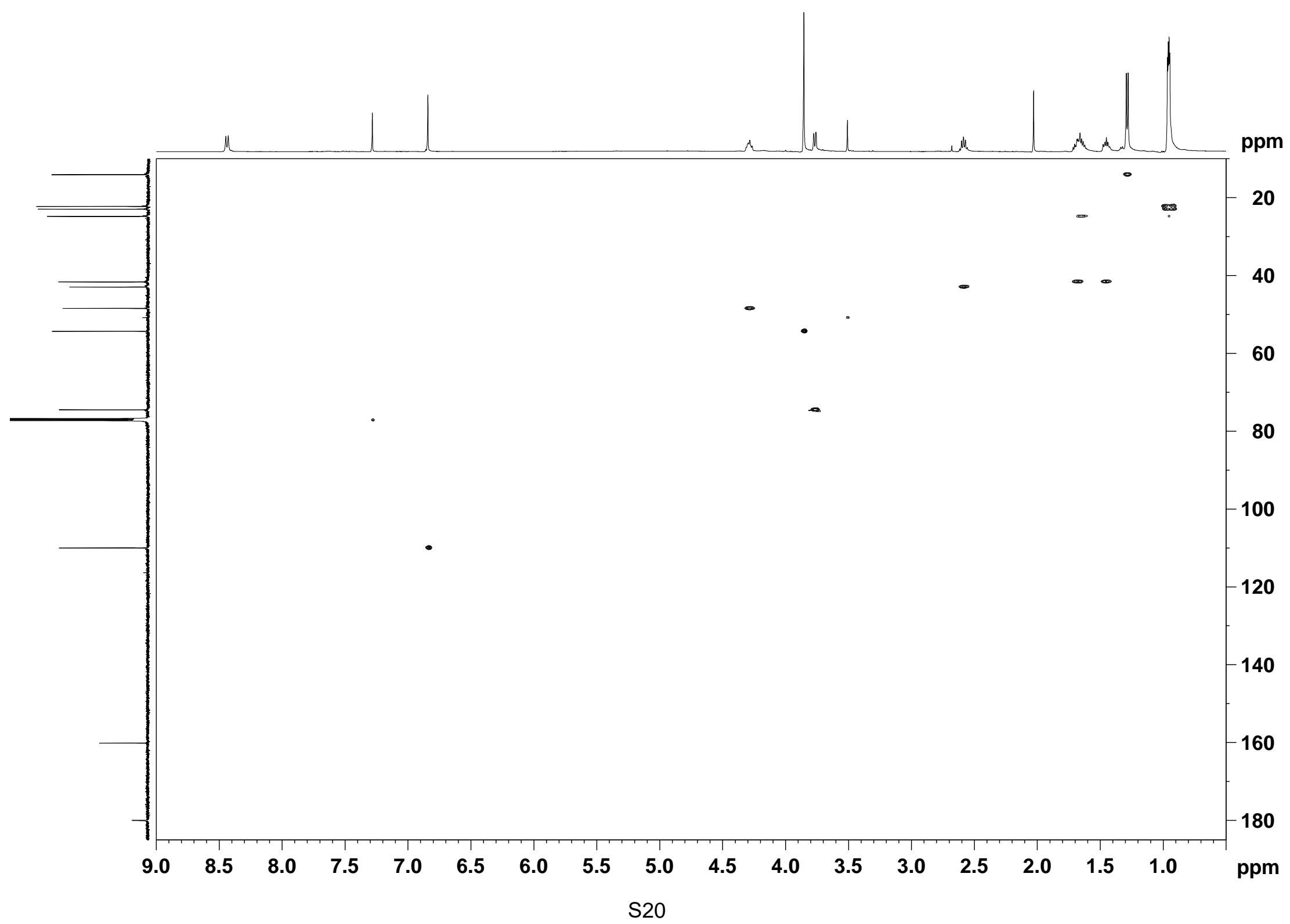


Figure S20. $\mathrm{HMBC}$ spectrum of $2\left(500 \mathrm{MHz}, \mathrm{CDCl}_{3}\right)$

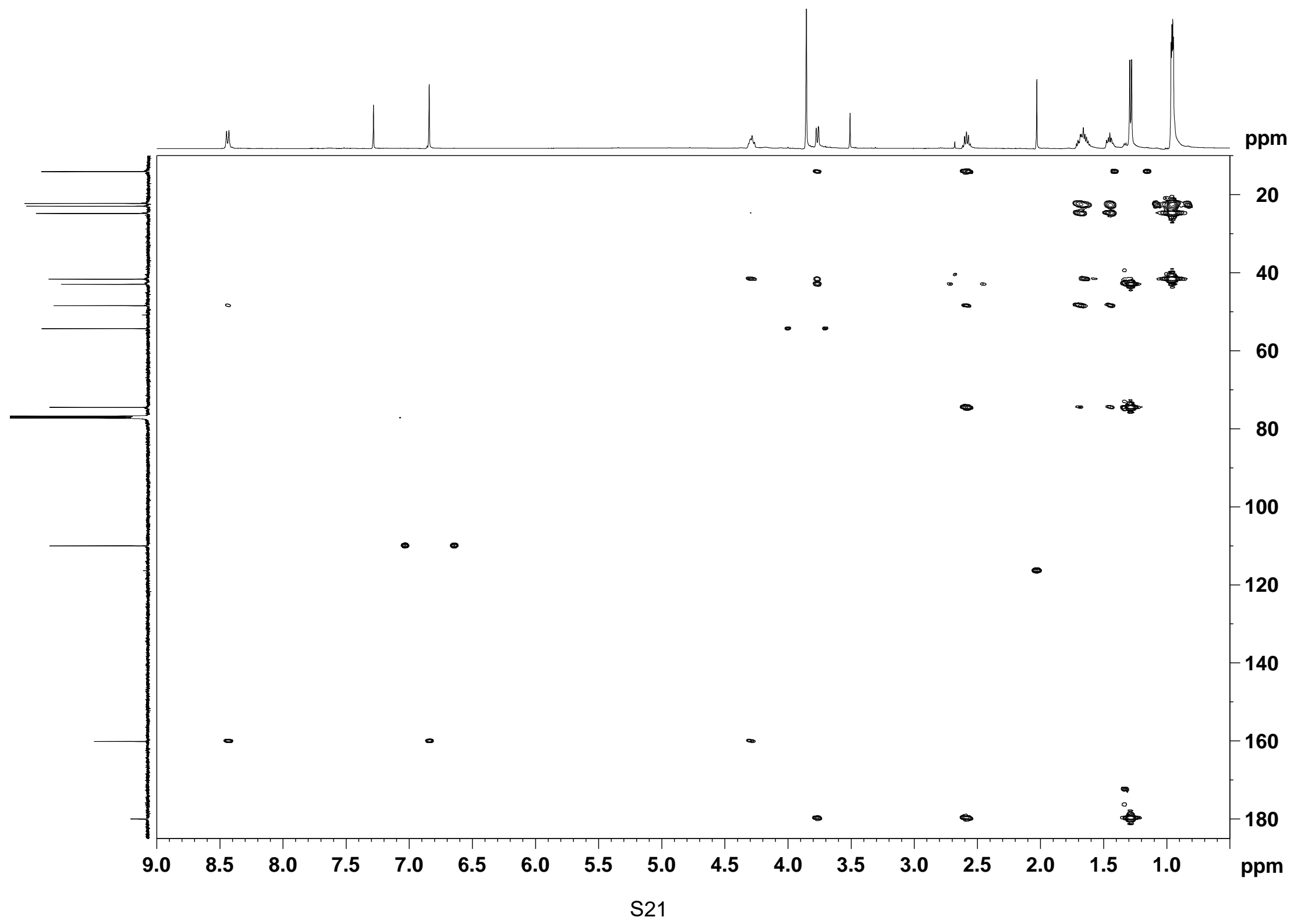


Figure S21. NOESY spectrum of $2\left(500 \mathrm{MHz}, \mathrm{CDCl}_{3}\right)$

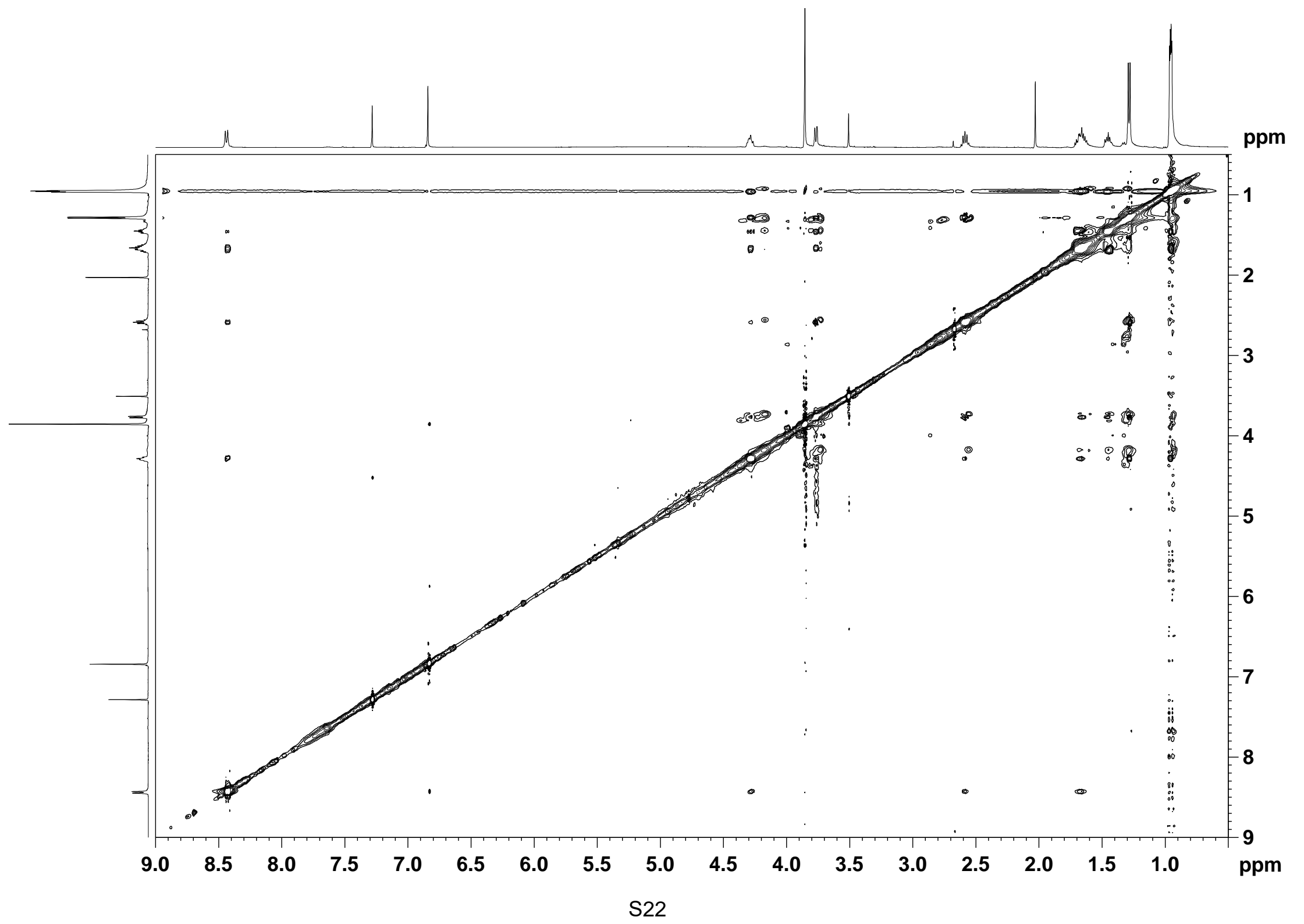


Figure S22. ${ }^{1} \mathrm{H}$ NMR spectrum of $\mathbf{3 a}\left(500 \mathrm{MHz}, \mathrm{CD}_{3} \mathrm{OD}\right)$

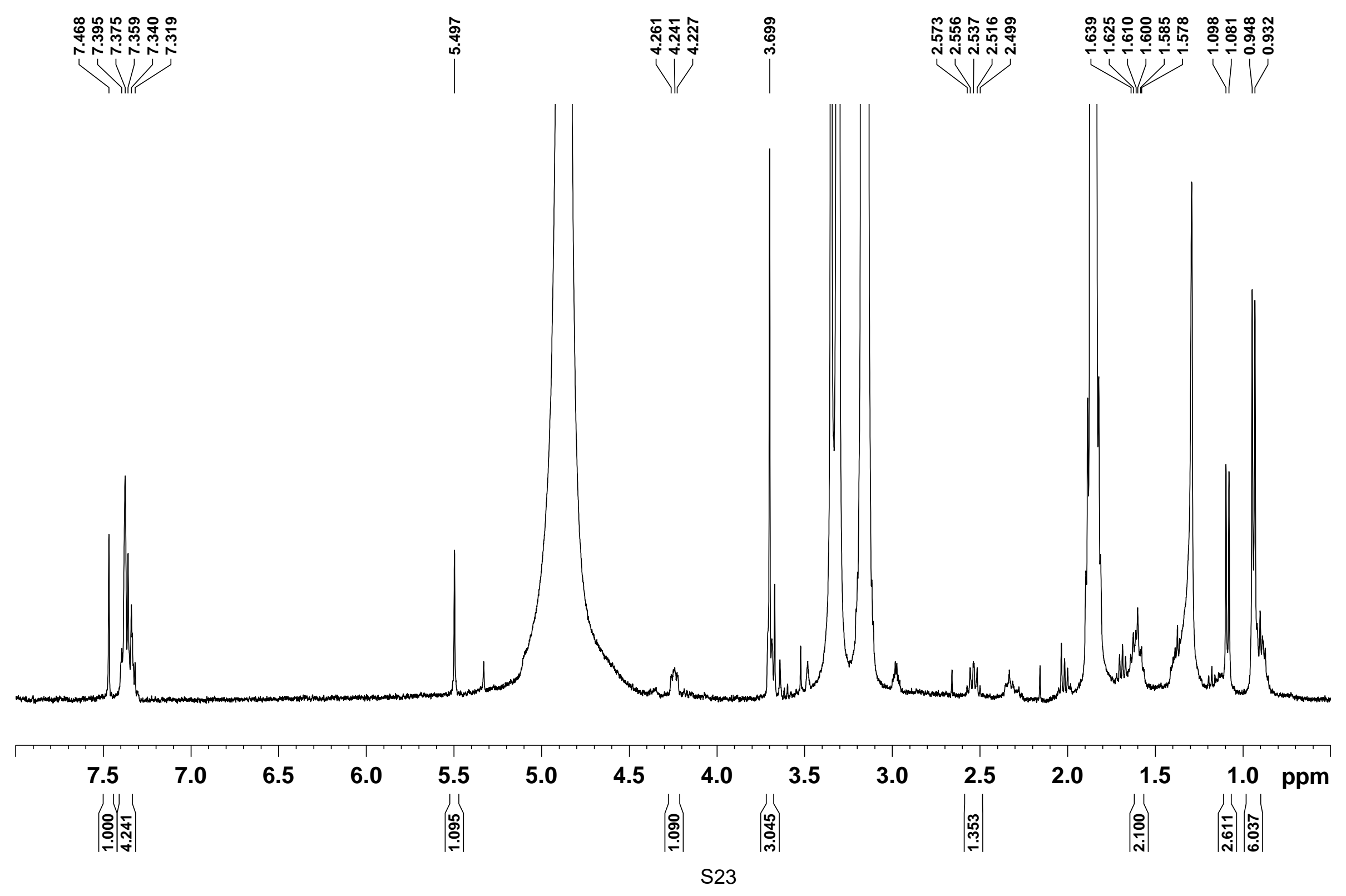


Figure S23. COSY spectrum of 3a (500 MHz, $\left.\mathrm{CD}_{3} \mathrm{OD}\right)$

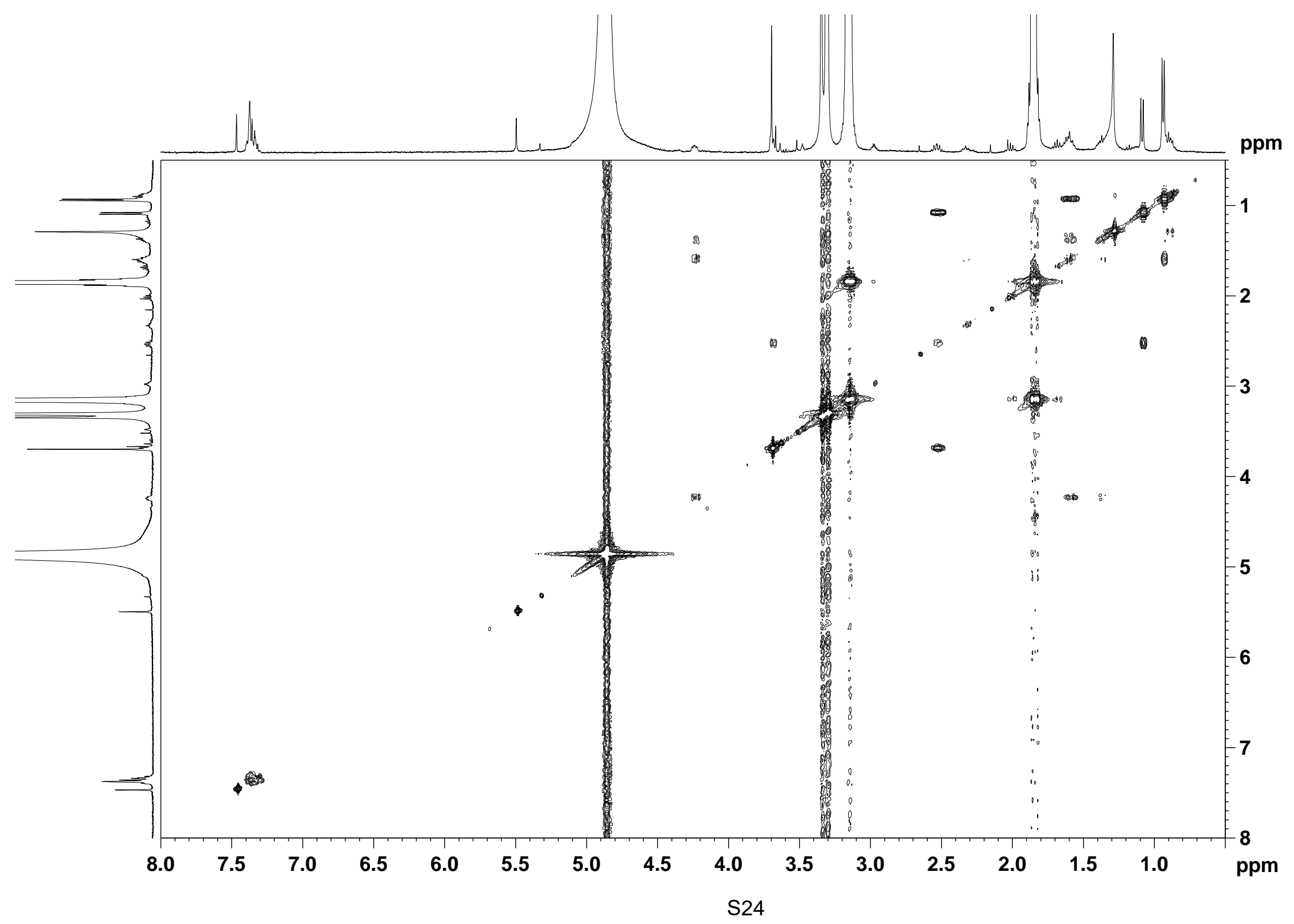


Figure S24. ${ }^{1} \mathrm{H}$ NMR spectrum of $\mathbf{3 b}\left(500 \mathrm{MHz}, \mathrm{CD}_{3} \mathrm{OD}\right)$

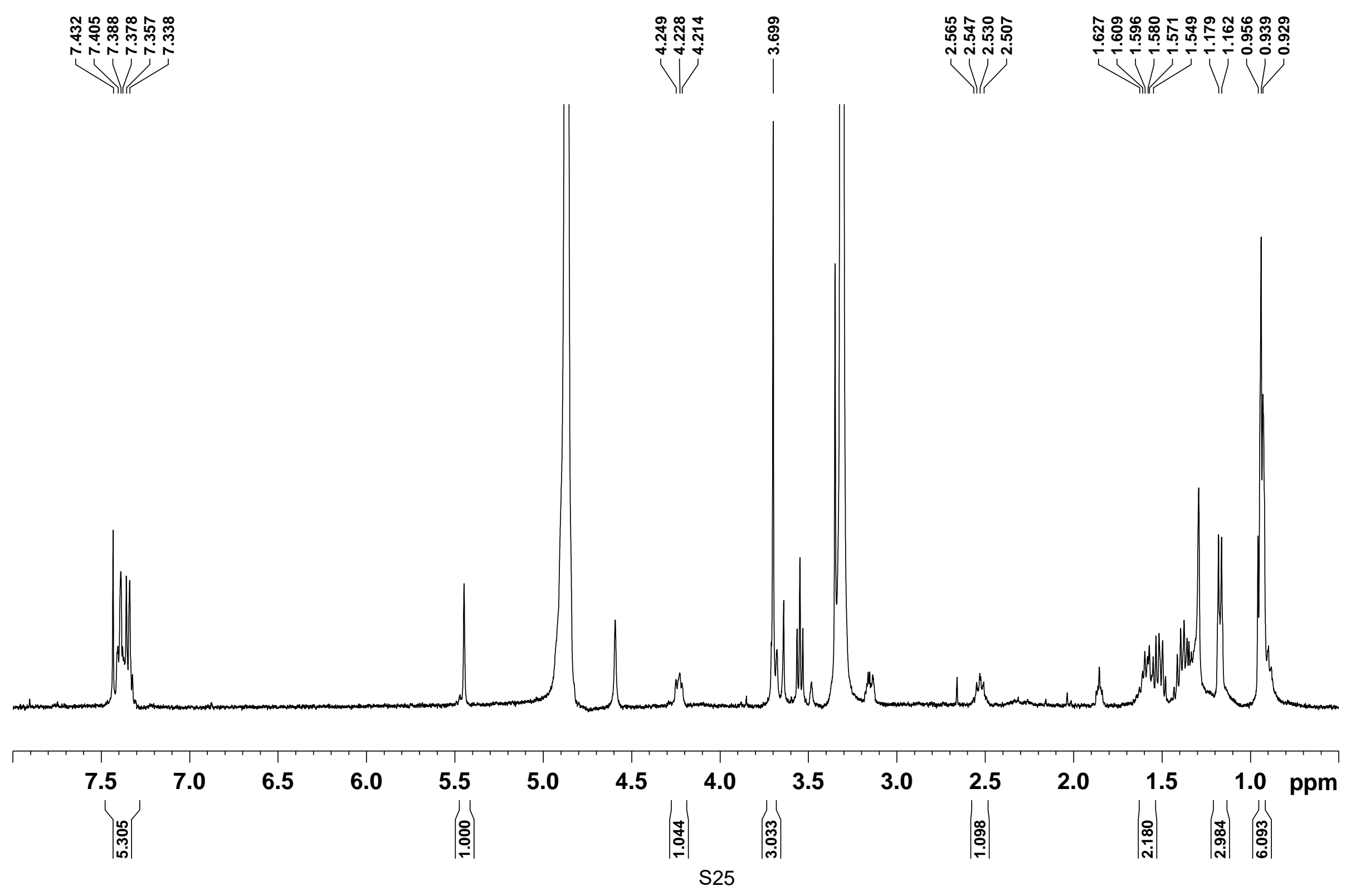


Figure S25. COSY spectrum of $\mathbf{3 b}\left(500 \mathrm{MHz}, \mathrm{CD}_{3} \mathrm{OD}\right)$

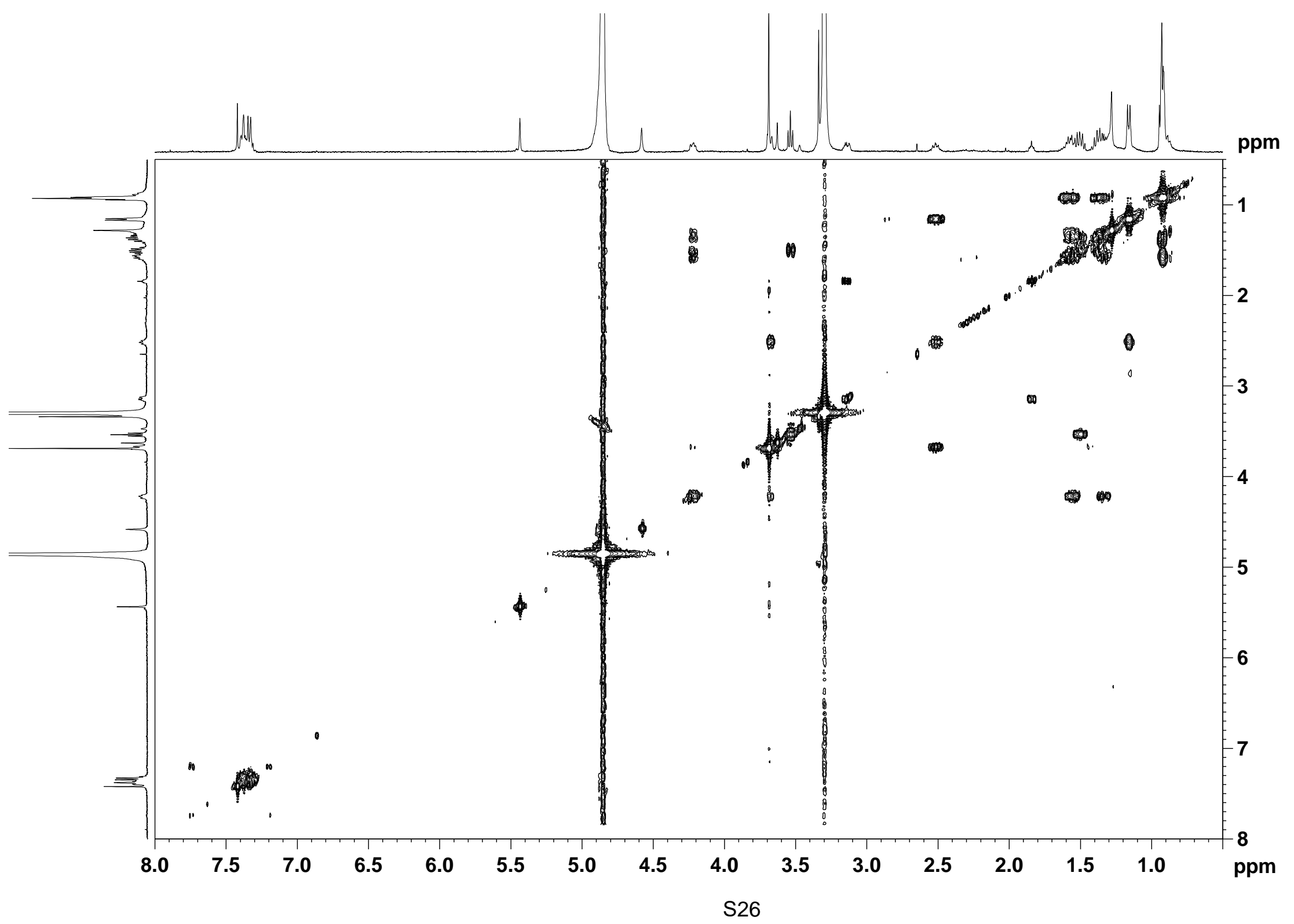


Figure S26. ${ }^{13} \mathrm{C}\left\{{ }^{1} \mathrm{H}\right\}$ NMR spectra of 1 labelled with DL-[2- $\left.{ }^{13} \mathrm{C}\right]$ leucine, $\left[1-{ }^{13} \mathrm{C}\right]$ propionate, $\left[1-{ }^{13} \mathrm{C}\right]$ acetate, and $\left[1-{ }^{13} \mathrm{C}\right]$ glycine $\left(125 \mathrm{MHz}\right.$, acetone- $\left.d_{6}\right)$

DL- $\left[2-{ }^{13} \mathrm{C}\right]$ leucine

$\begin{array}{llllllllllllllllllllll}180 & 170 & 160 & 150 & 140 & 130 & 120 & 110 & 100 & 90 & 80 & 70 & 60 & 50 & 40 & 30 & 20 & \text { ppm }\end{array}$

$\left[1-{ }^{13}\right.$ C]propionate

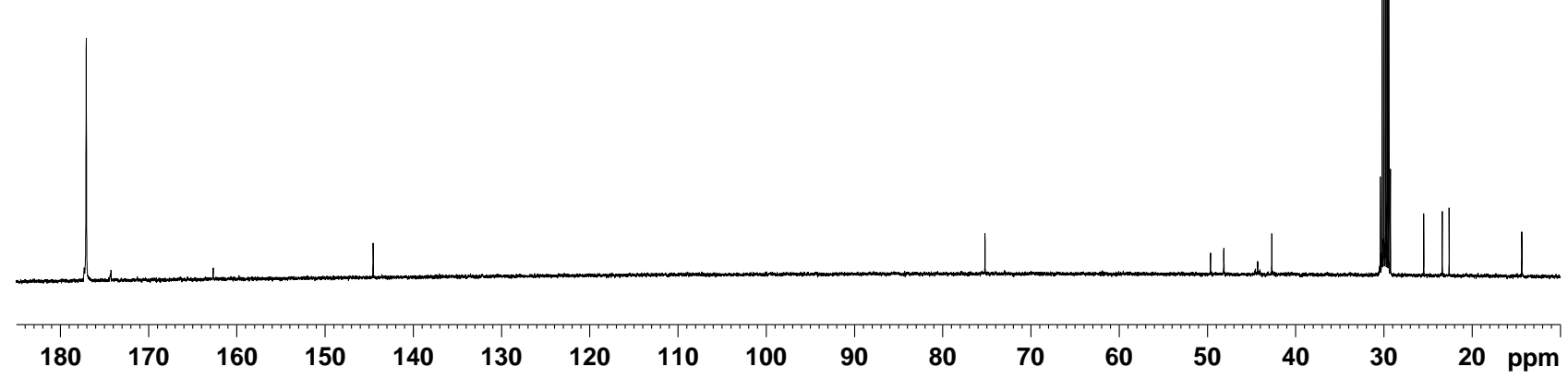

$\left[1-{ }^{13} \mathrm{C}\right]$ acetate

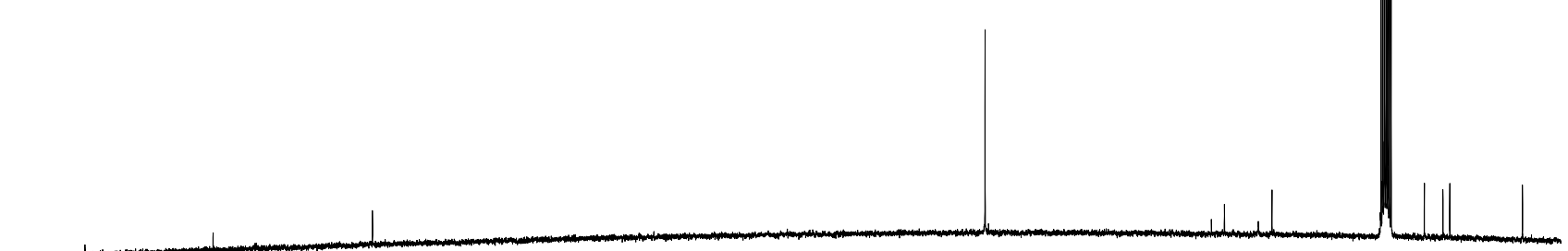

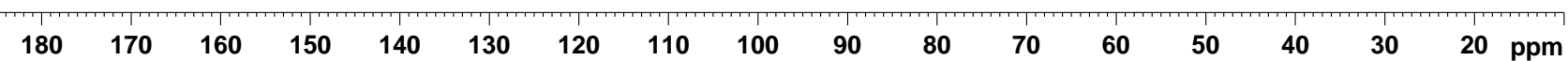

$\left[1-{ }^{13} \mathrm{C}\right] \mathrm{glycine}$

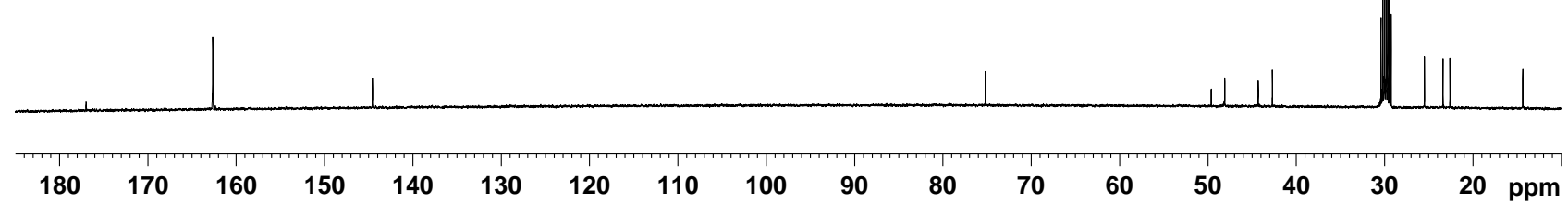

control (no feeding)

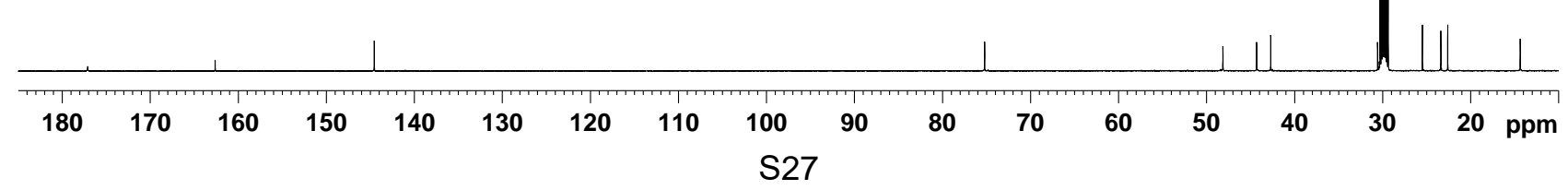


Figure S27. ${ }^{13} \mathrm{C}\left\{{ }^{1} \mathrm{H}\right\}$ NMR spectra of 2 labelled with DL- $\left[2-{ }^{13} \mathrm{C}\right]$ leucine, $\left[1-{ }^{13} \mathrm{C}\right]$ propionate, $\left[1-{ }^{13} \mathrm{C}\right]$ acetate, and $\left[1-{ }^{13} \mathrm{C}\right]$ glycine $\left(125 \mathrm{MHz}, \mathrm{CDCl}_{3}\right)$

DL- $\left[2-{ }^{13} \mathrm{C}\right]$ leucine

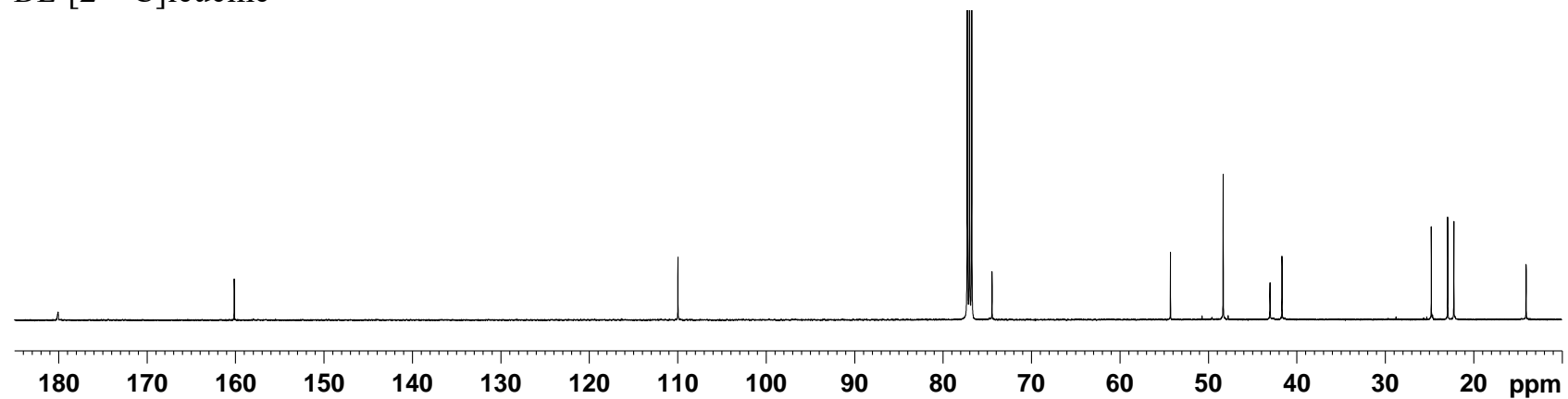

$\left[1-{ }^{13}\right.$ C]propionate

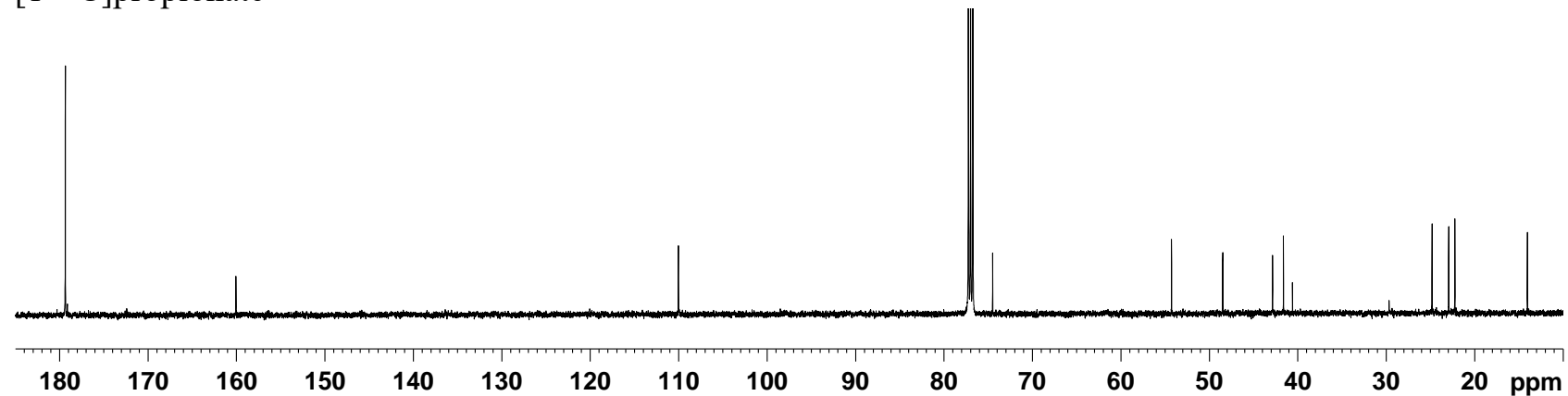

$\left[1-{ }^{13} \mathrm{C}\right]$ acetate

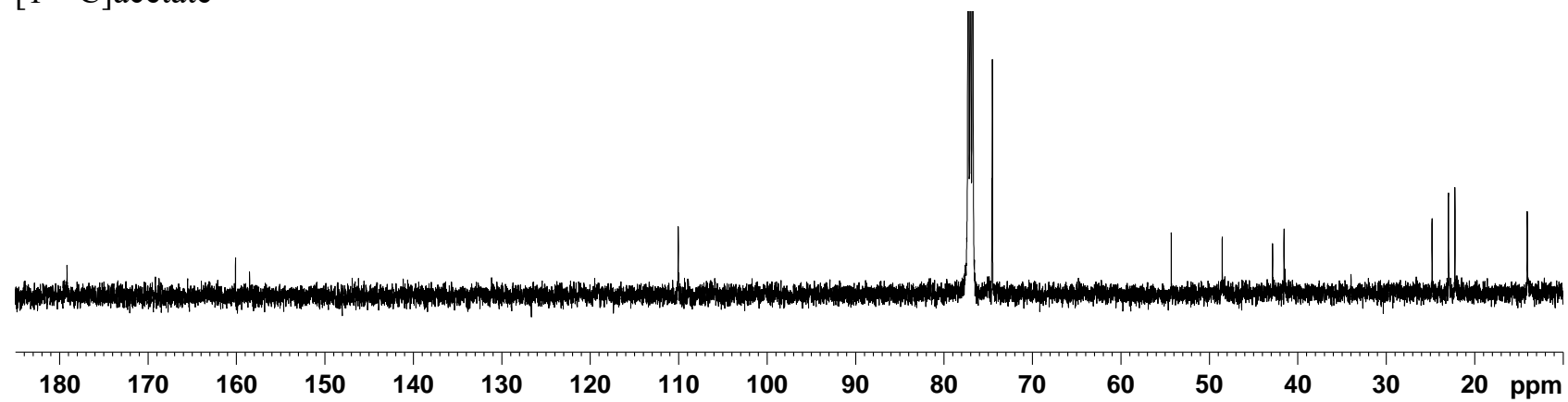

$\left[1-{ }^{13} \mathrm{C}\right]$ glycine

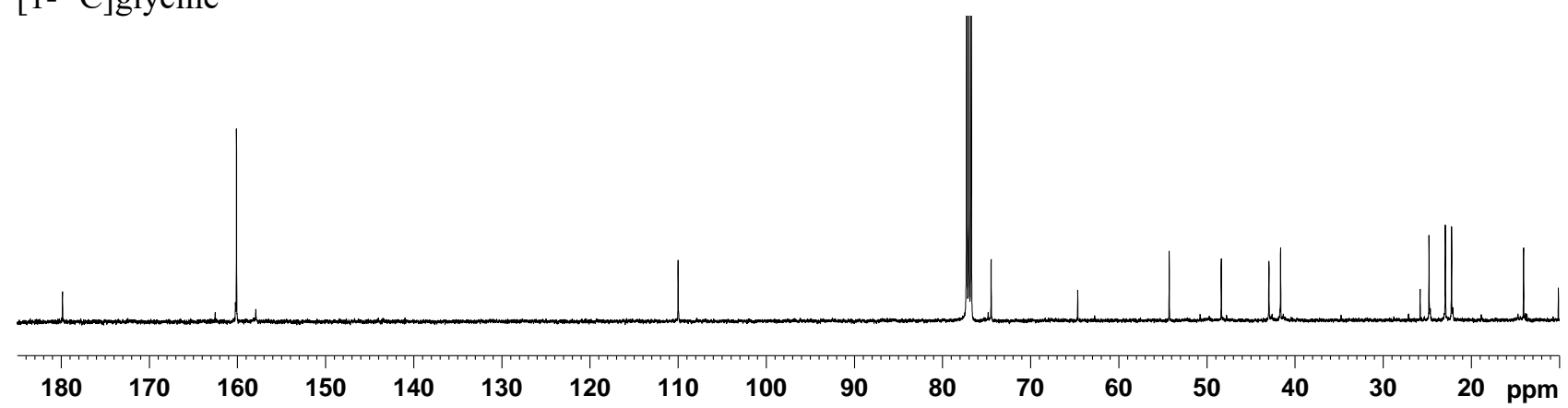

control (no feeding)

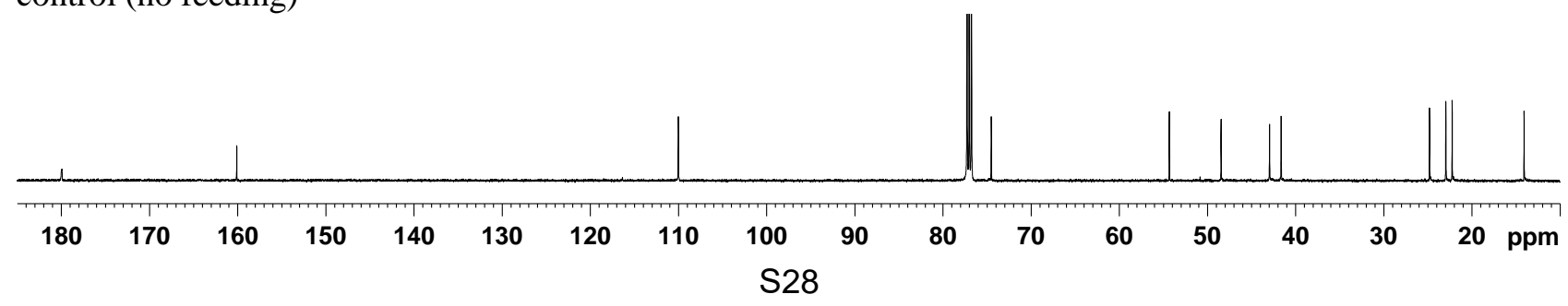


Figure S28. ${ }^{1} \mathrm{H}$ NMR spectra of $9\left(500 \mathrm{MHz}, \mathrm{C}_{6} \mathrm{D}_{6}, 343 \mathrm{~K}\right)$

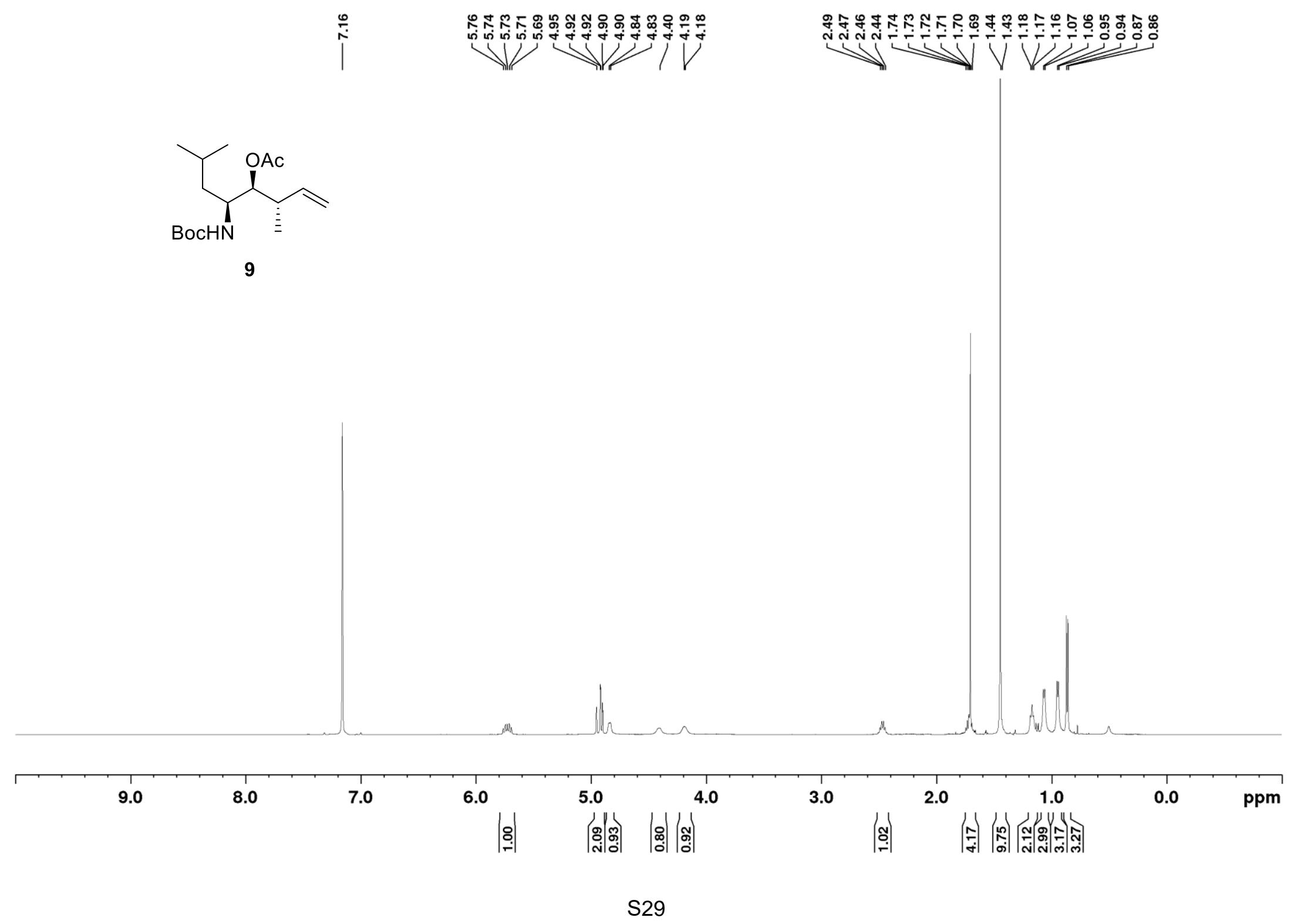


Figure S29. ${ }^{13} \mathrm{C}\left\{{ }^{1} \mathrm{H}\right\}$ NMR spectra of $9\left(125 \mathrm{MHz}, \mathrm{C}_{6} \mathrm{D}_{6}, 336 \mathrm{~K}\right)$

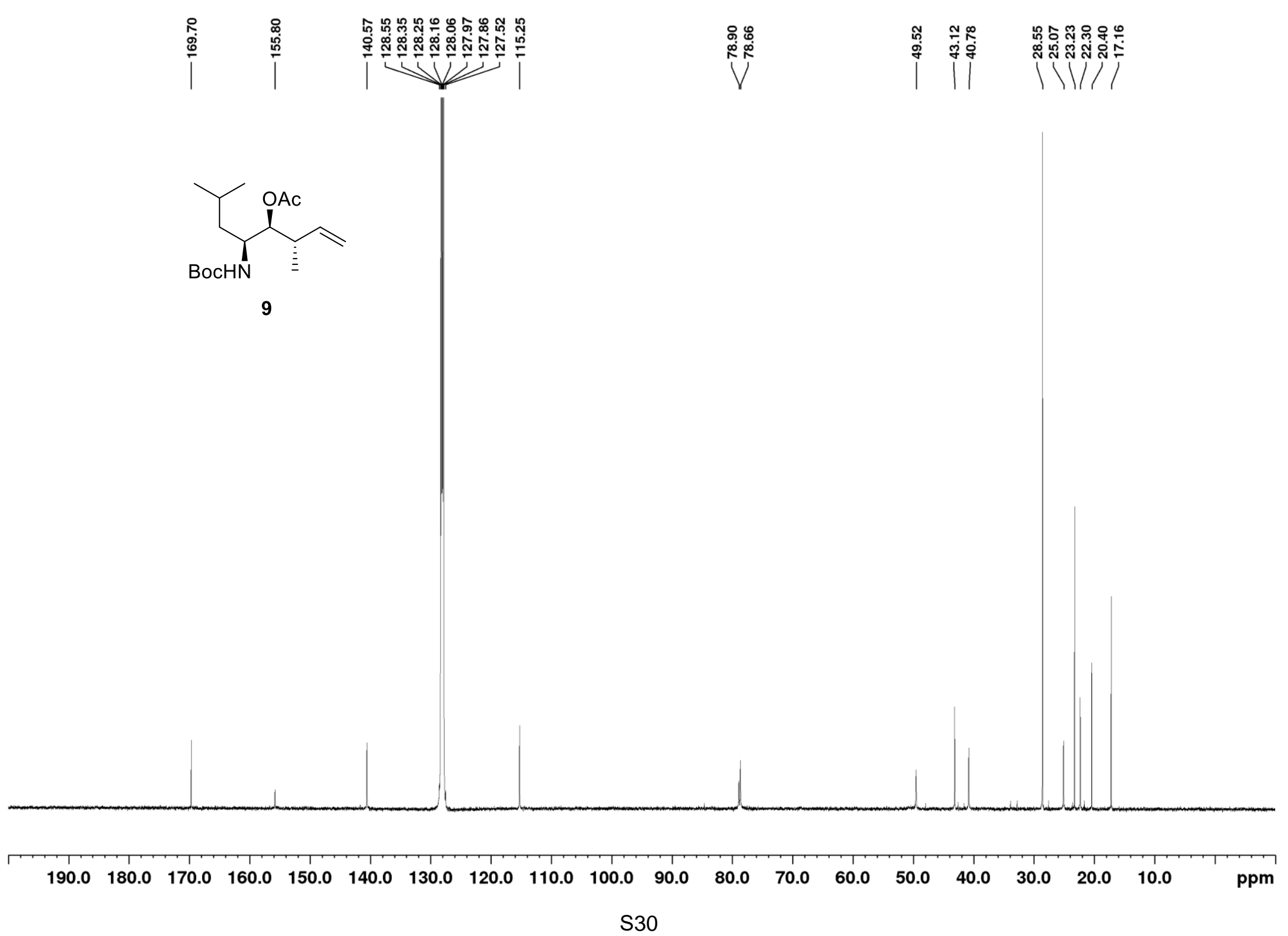


Figure S30. ${ }^{1} \mathrm{H}$ NMR spectra of $\mathbf{1 1}\left(400 \mathrm{MHz}, \mathrm{CDCl}_{3}\right)$

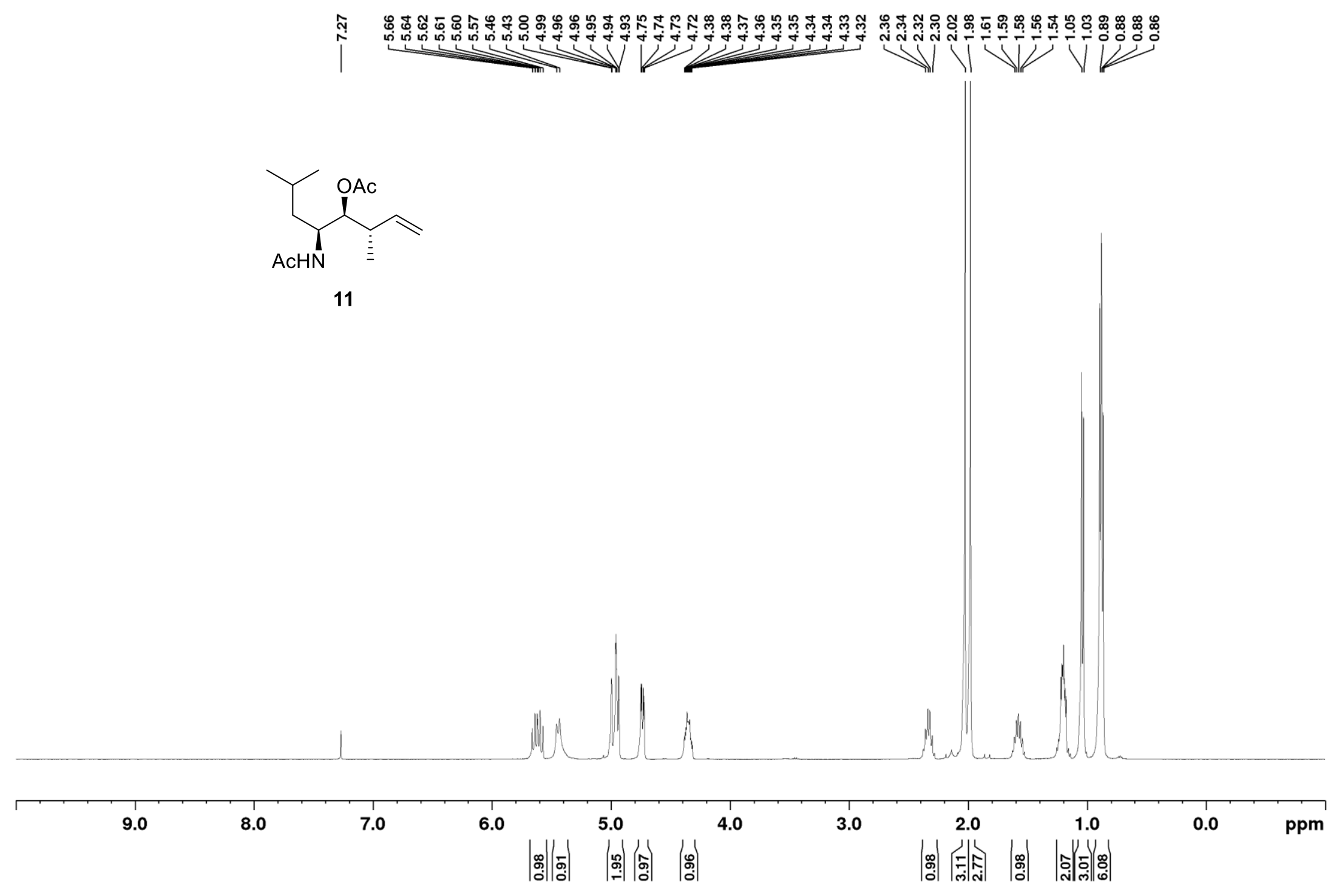


Figure S31. ${ }^{13} \mathrm{C}\left\{{ }^{1} \mathrm{H}\right\}$ NMR spectra of $\mathbf{1 1}\left(100 \mathrm{MHz}, \mathrm{CDCl}_{3}\right)$

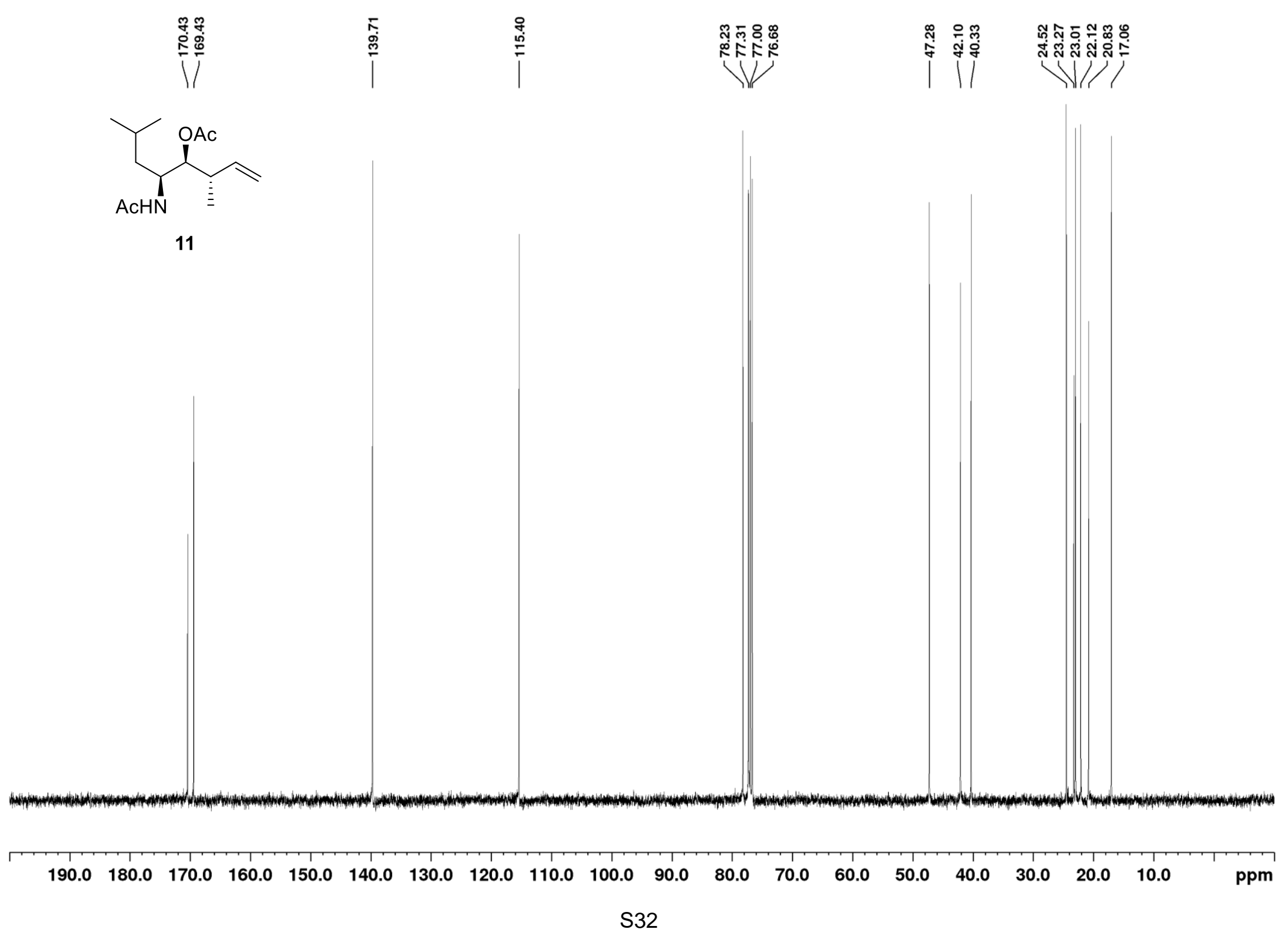


Figure S32. ${ }^{1} \mathrm{H}$ NMR spectra of $\mathbf{1 4}\left(500 \mathrm{MHz}, \mathrm{C}_{6} \mathrm{D}_{6}, 333 \mathrm{~K}\right)$

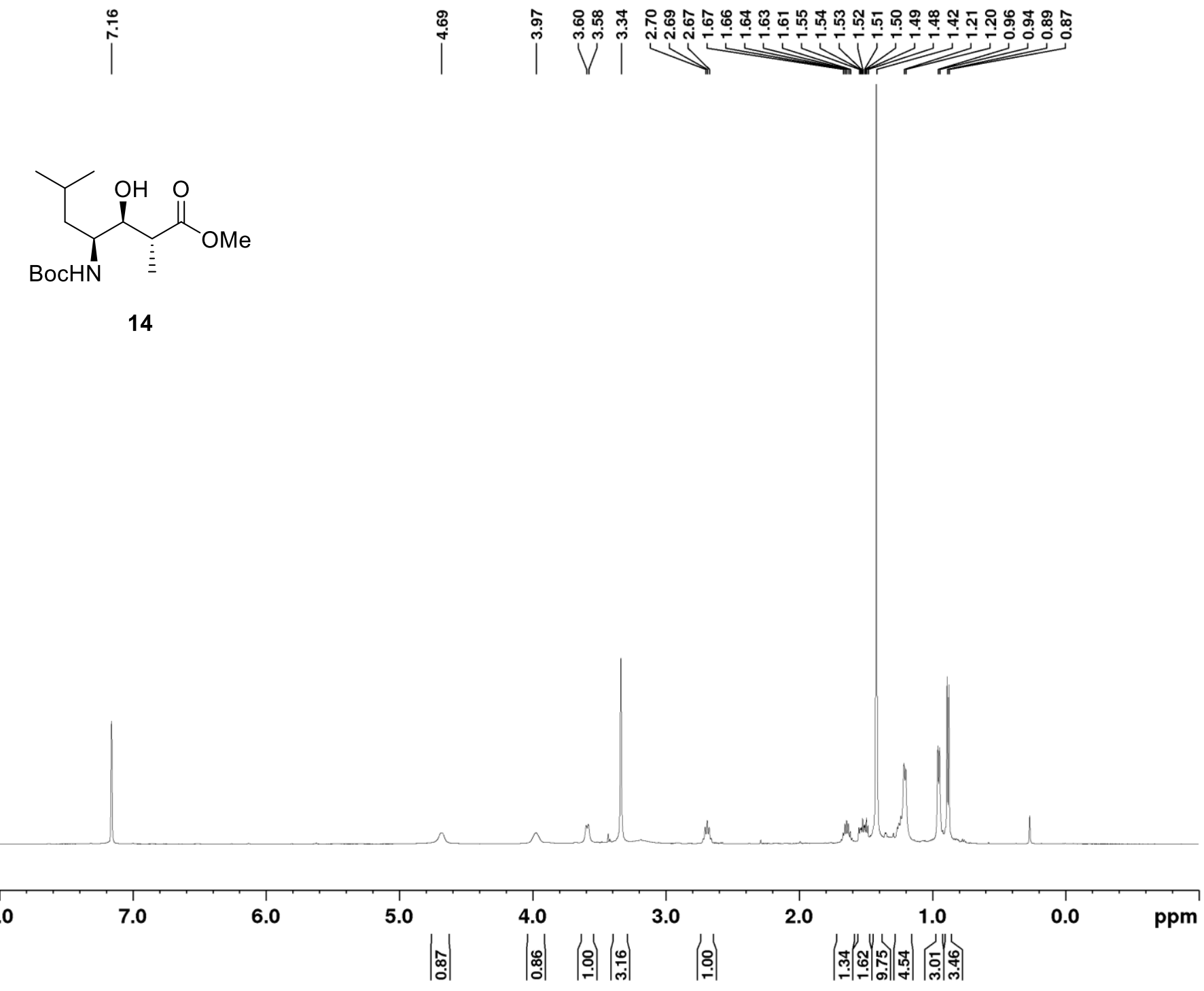


Figure S33. ${ }^{13} \mathrm{C}\left\{{ }^{1} \mathrm{H}\right\}$ NMR spectra of $\mathbf{1 4}\left(125 \mathrm{MHz}, \mathrm{C}_{6} \mathrm{D}_{6}, 333 \mathrm{~K}\right)$

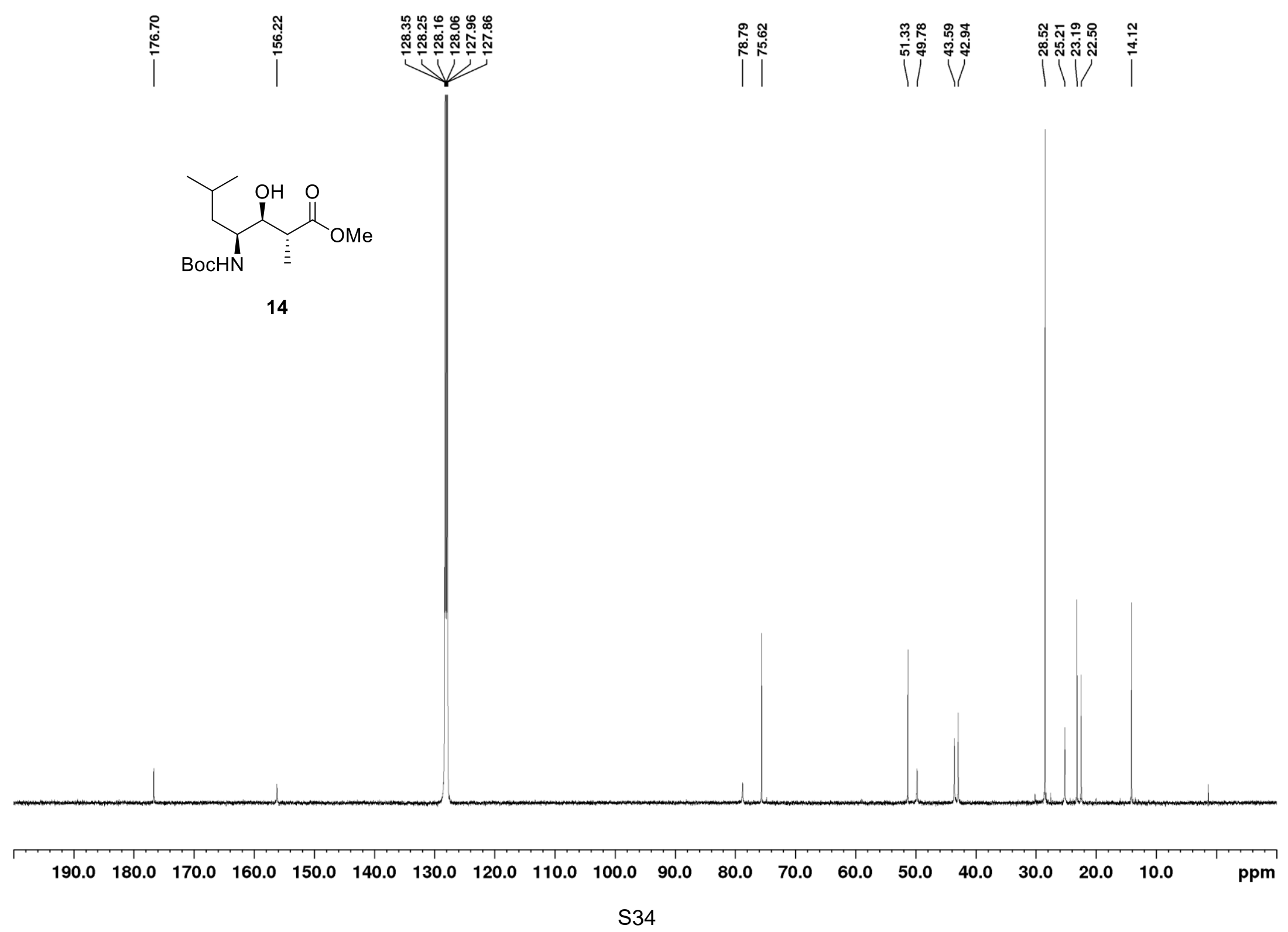


Figure S34. ${ }^{1} \mathrm{H}$ NMR spectra of $\mathbf{1 5}\left(400 \mathrm{MHz}, \mathrm{CD}_{3} \mathrm{OD}\right)$

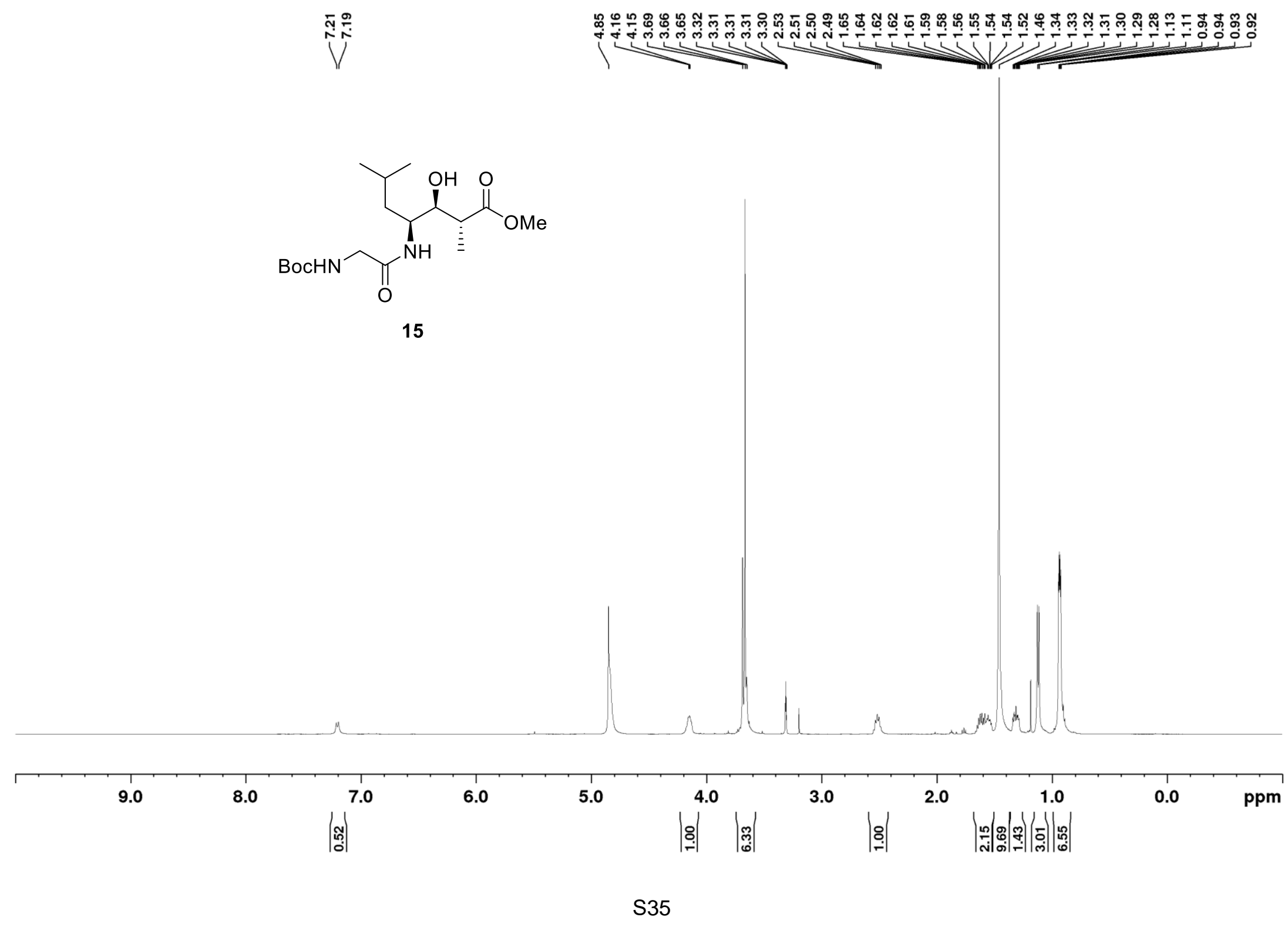


Figure S35. ${ }^{13} \mathrm{C}\left\{{ }^{1} \mathrm{H}\right\}$ NMR spectra of $\mathbf{1 5}\left(125 \mathrm{MHz}, \mathrm{CD}_{3} \mathrm{OD}\right)$

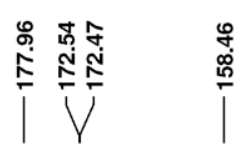

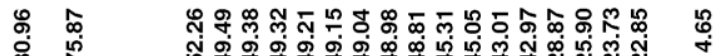

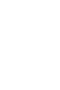

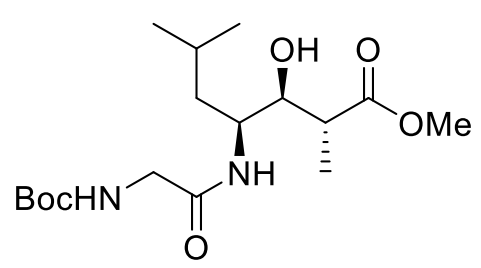

15

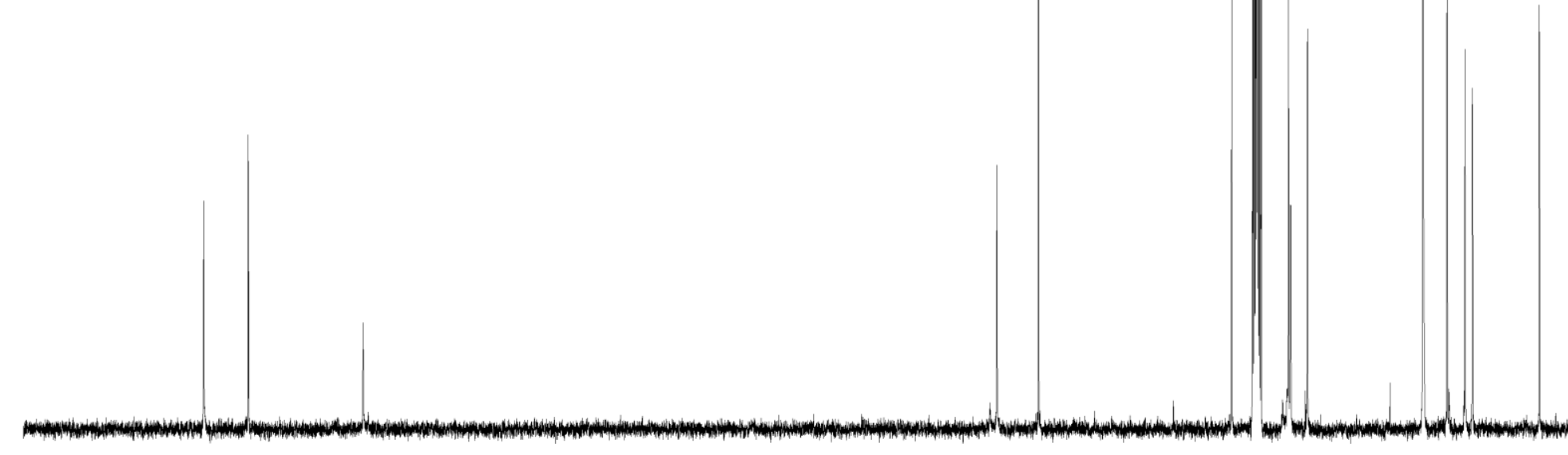

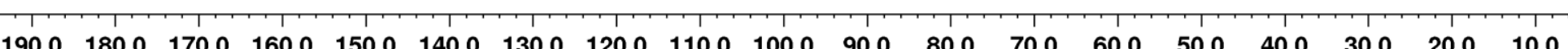
ppm 
Figure S36. ${ }^{1} \mathrm{H}$ NMR spectra of synthetic $1\left(500 \mathrm{MHz}, \mathrm{CD}_{3} \mathrm{OD}\right)$

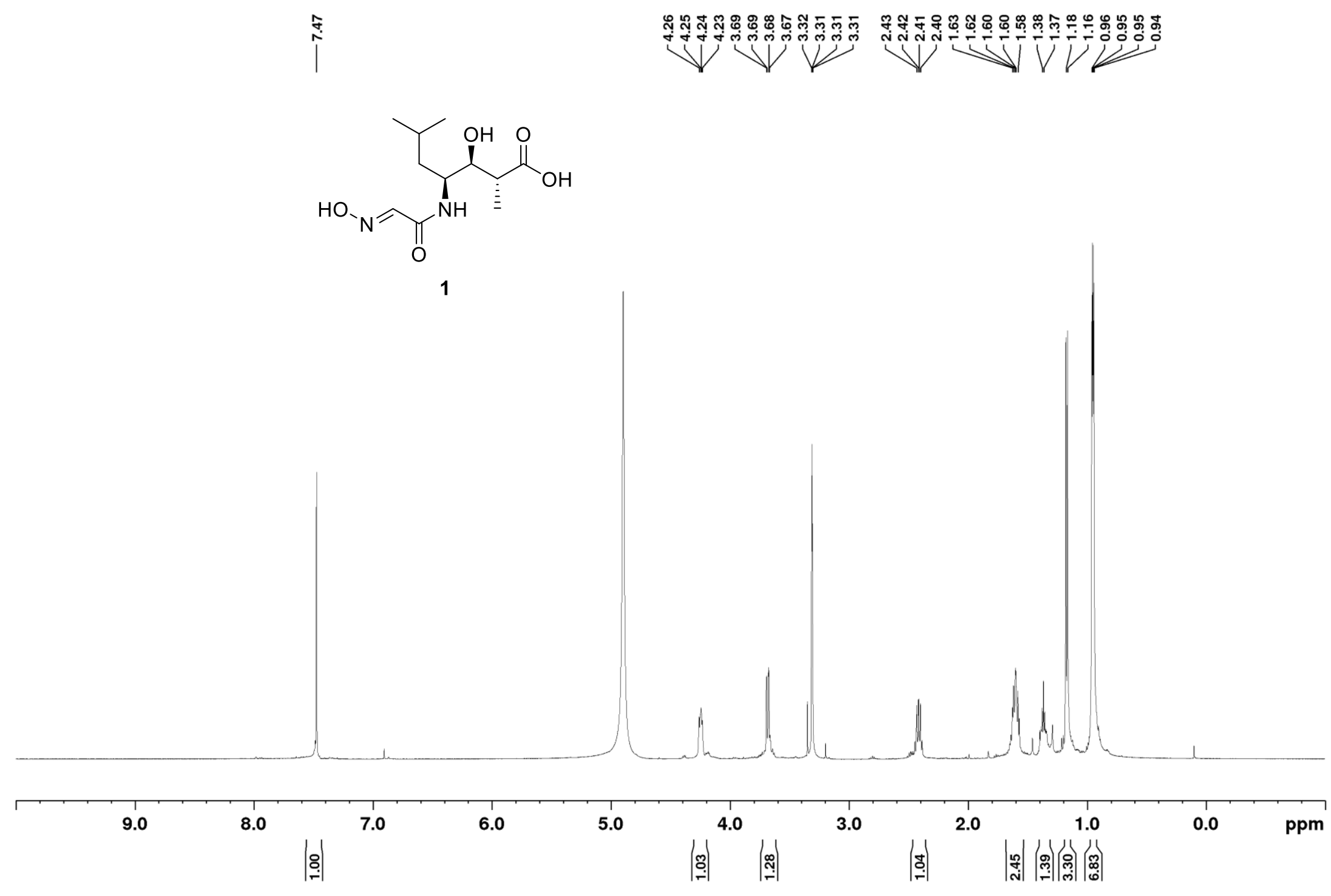


Figure S37. ${ }^{13} \mathrm{C}\left\{{ }^{1} \mathrm{H}\right\}$ NMR spectra of synthetic $\mathbf{1}\left(125 \mathrm{MHz}, \mathrm{CD}_{3} \mathrm{OD}\right)$

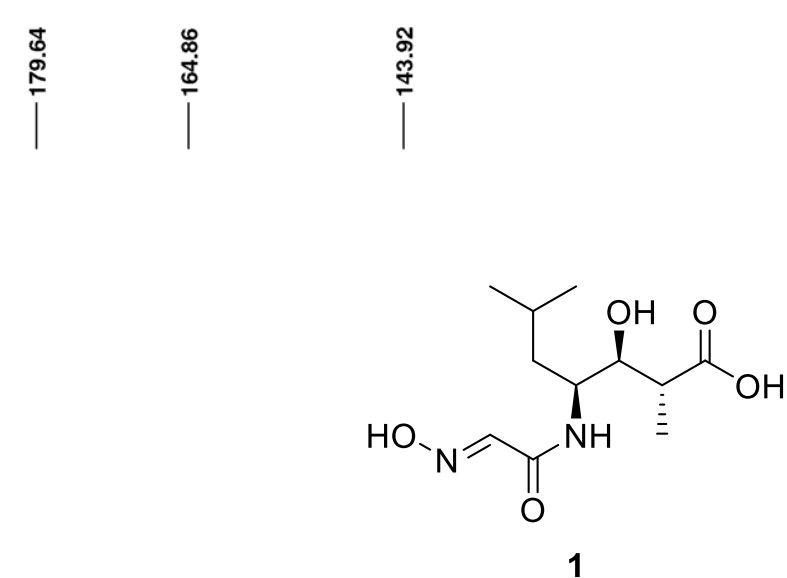

帝

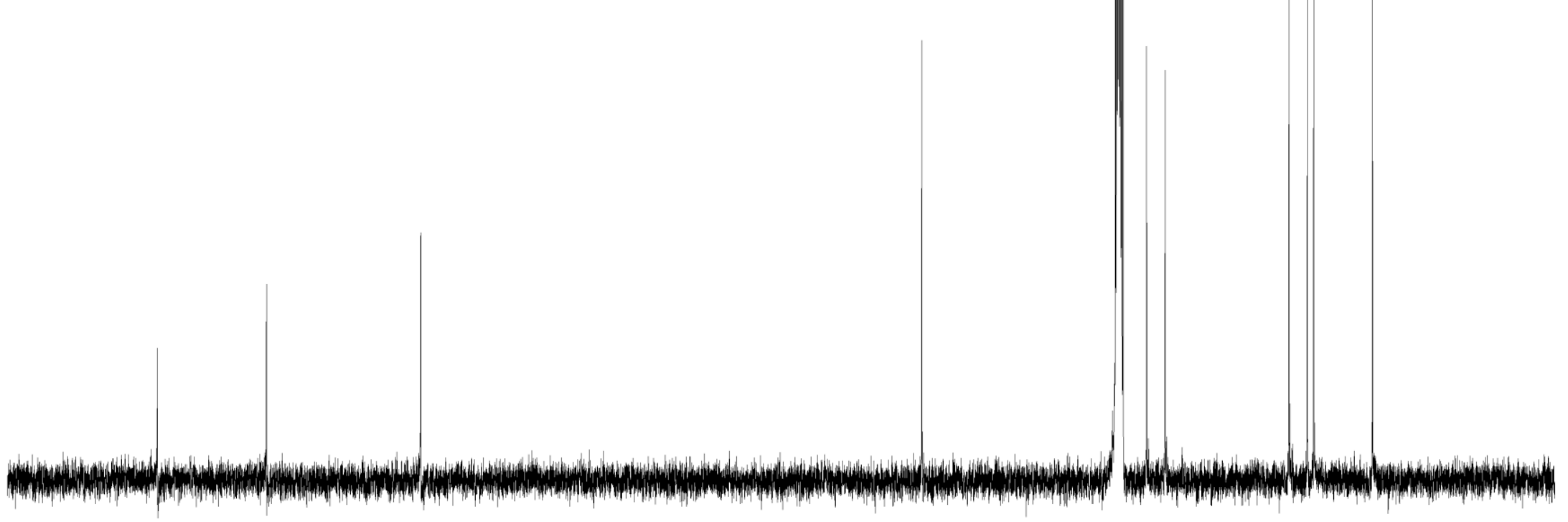

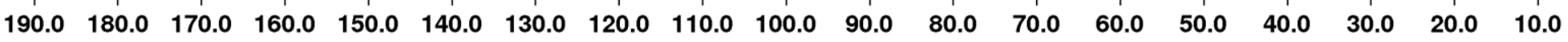
ppm 
Figure S38. ${ }^{1} \mathrm{H}$ NMR spectra of $17\left(500 \mathrm{MHz}, \mathrm{CD}_{3} \mathrm{OD}\right)$

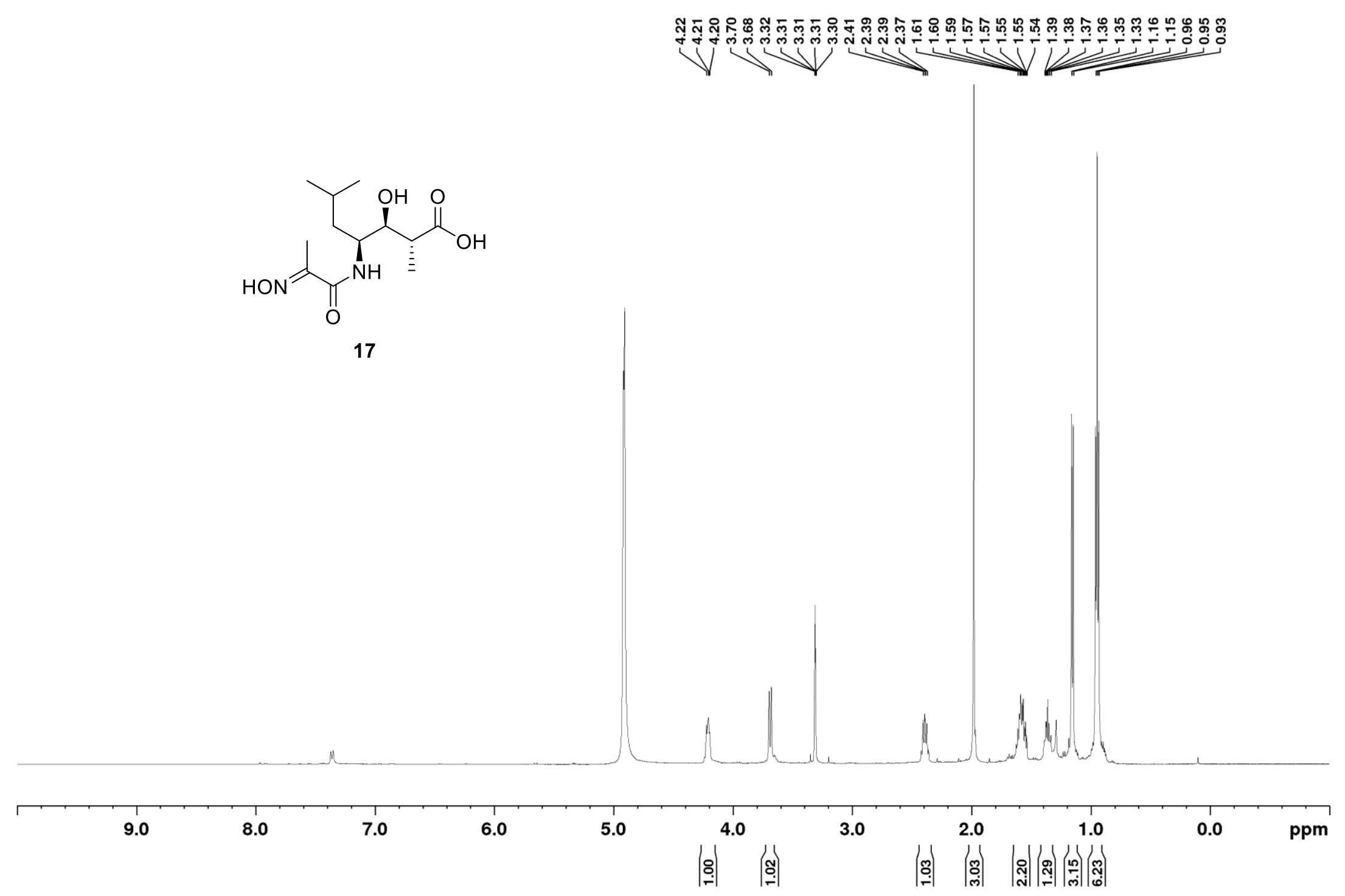


Figure S39. ${ }^{13} \mathrm{C}\left\{{ }^{1} \mathrm{H}\right\}$ NMR spectra of $\mathbf{1 7}\left(125 \mathrm{MHz}, \mathrm{CD}_{3} \mathrm{OD}\right)$

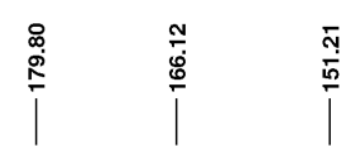

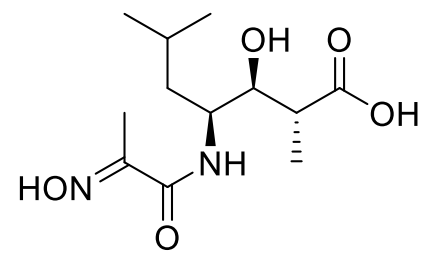

17

|

$+\sqrt{1}$

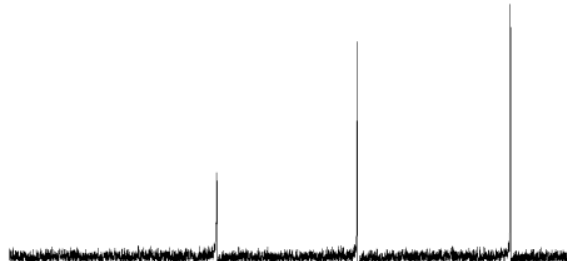


Figure S40. ${ }^{1} \mathrm{H}$ NMR spectra of $\mathbf{1 8}\left(400 \mathrm{MHz}, \mathrm{CD}_{3} \mathrm{OD}\right)$
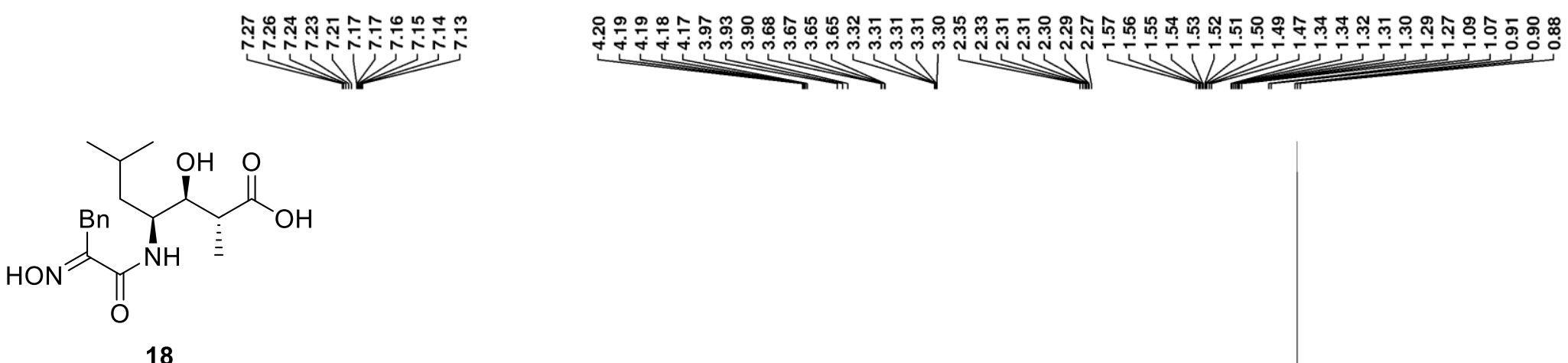

18

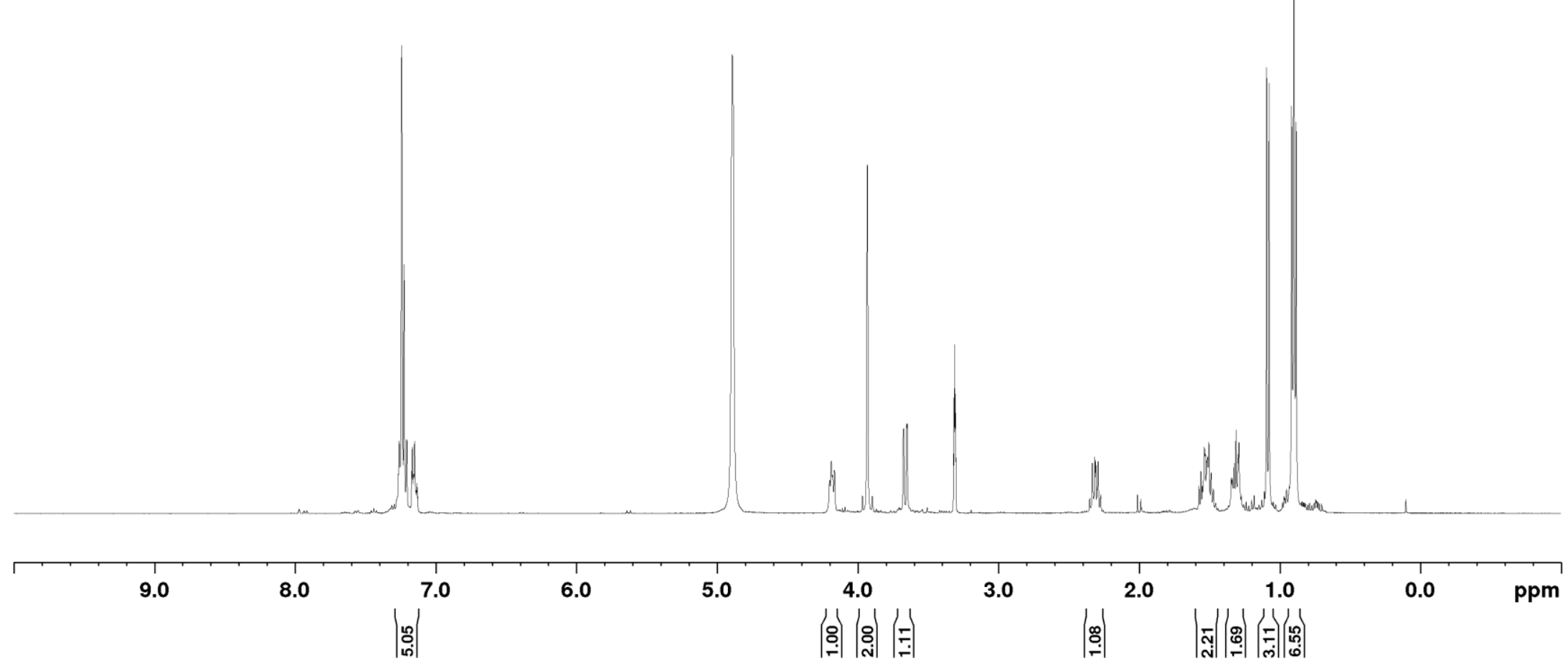


Figure S41. ${ }^{13} \mathrm{C}\left\{{ }^{1} \mathrm{H}\right\}$ NMR spectra of $18\left(100 \mathrm{MHz}, \mathrm{CD}_{3} \mathrm{OD}\right)$

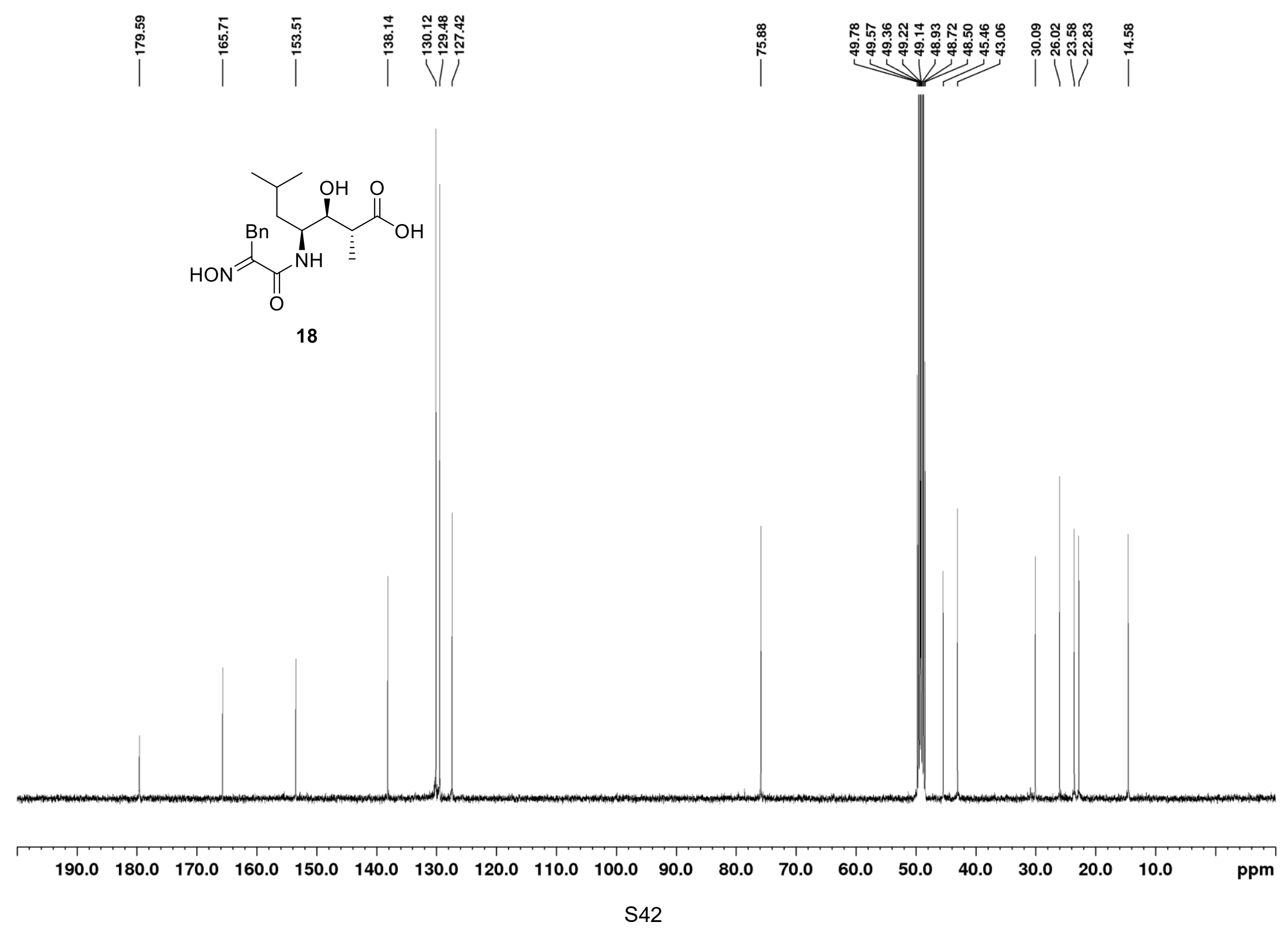


Figure S42. Comparison of ${ }^{1} \mathrm{H}$ NMR spectra of natural and synthetic $1\left(500 \mathrm{MHz}, \mathrm{CD}_{3} \mathrm{OD}\right)$

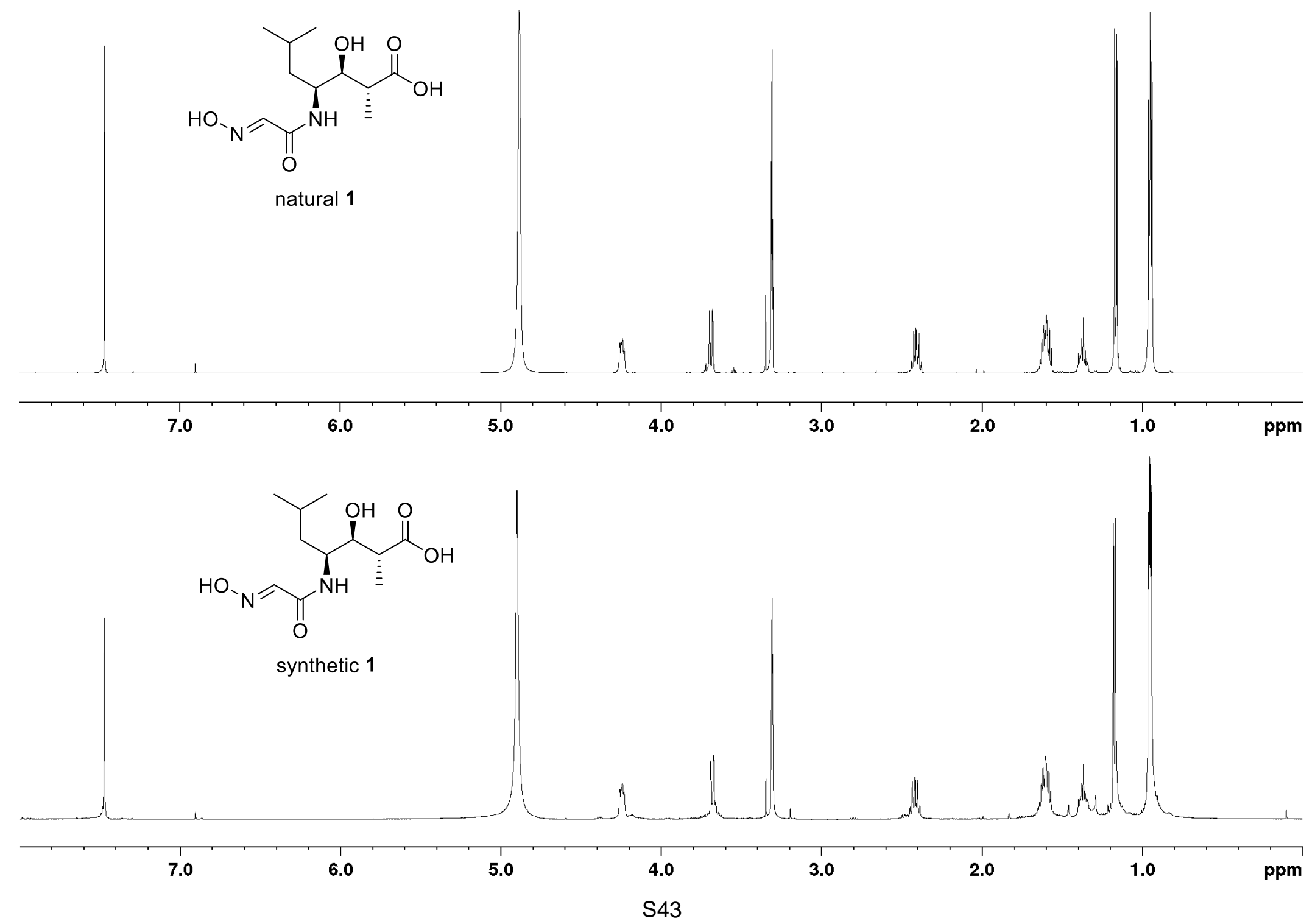


Figure S43. Comparison of ${ }^{13} \mathrm{C}\left\{{ }^{1} \mathrm{H}\right\}$ NMR spectra of natural and synthetic $1\left(125 \mathrm{MHz}, \mathrm{CD}_{3} \mathrm{OD}\right)$

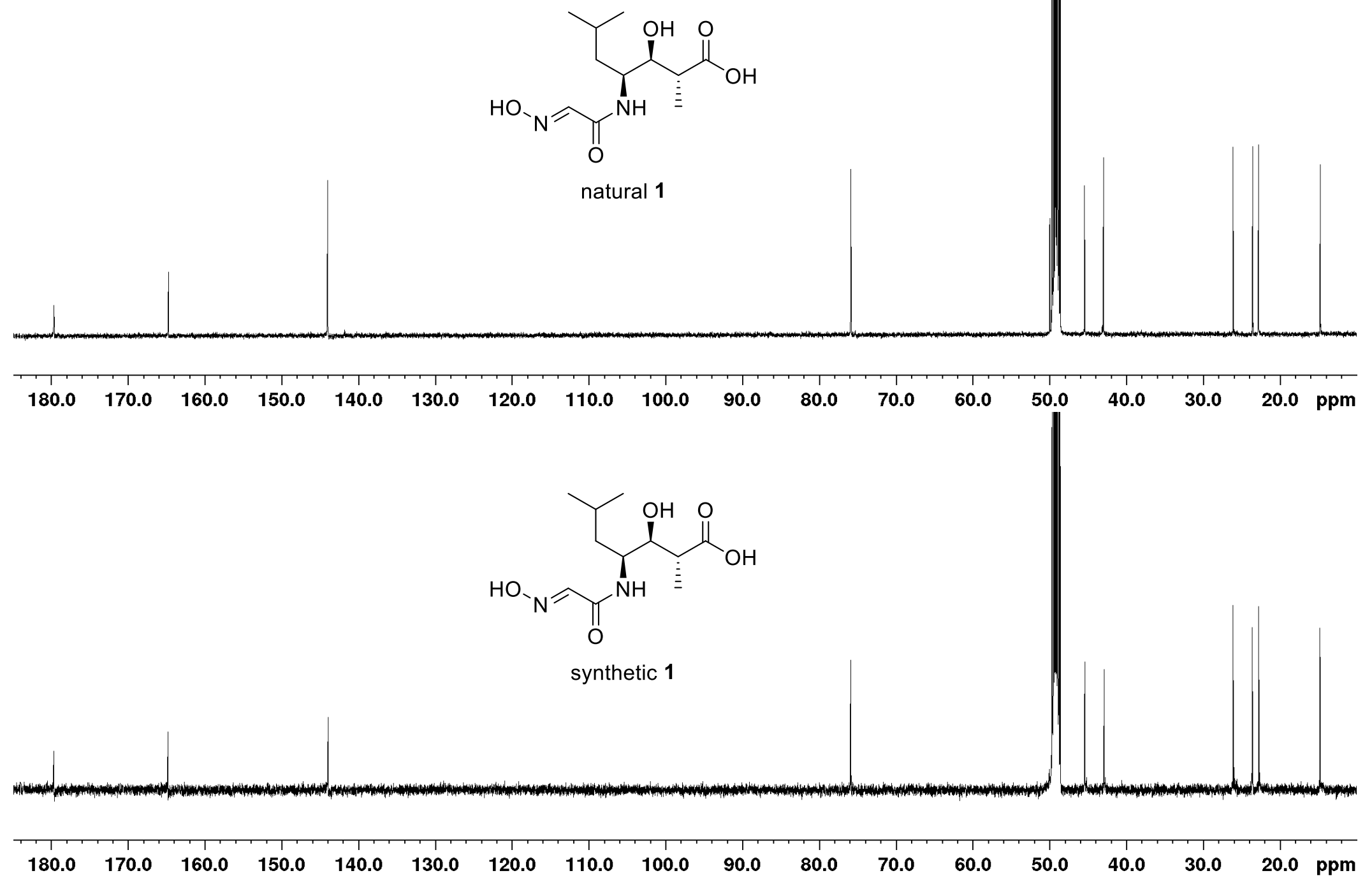


Table S1. Structure, energy and Cartesian coordinate of 2 a

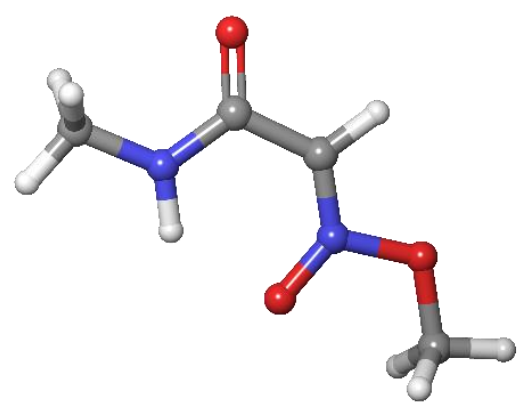

$\mathbf{2 a}$ (no imaginary frequencies)

B3LYP/6-311+G(d,p)-IEFPCM $\left(\mathrm{CHCl}_{3}\right) / / B 3 L Y P / 6-31+G(d, p)$ :

Gibbs Free Energy (a.u.) $=-492.376247$

B3LYP/6-311+G(d,p)-IEFPCM $\left(\mathrm{CHCl}_{3}\right)$ :

Electronic energy (a.u.) $=-492.471940$

B3LYP/6-31+G(d,p):

Zero-point correction (a.u.) $=0.132753$

Thermal correction to Energy (a.u.) $=0.143047$

Thermal correction to Enthalpy (a.u.) $=0.143991$

Thermal correction to Gibbs Free Energy (a.u.) $=0.095693$

$\begin{array}{lrrr}\mathrm{N} & 1.71974 & -0.67616 & -0.00021 \\ \mathrm{C} & 1.33595 & 0.62530 & -0.00004 \\ \mathrm{O} & 2.13027 & 1.57101 & 0.00009 \\ \mathrm{C} & 3.12909 & -1.03585 & 0.00006 \\ \mathrm{C} & -0.11610 & 0.92951 & -0.00008 \\ \mathrm{~N} & -1.07458 & 0.03908 & 0.00000 \\ \mathrm{O} & -2.37371 & 0.62932 & 0.00004 \\ \mathrm{C} & -3.42687 & -0.34889 & -0.00001 \\ \mathrm{O} & -1.00661 & -1.20869 & 0.00007 \\ \mathrm{H} & 0.99962 & -1.38775 & 0.00008 \\ \mathrm{H} & 3.20926 & -2.12421 & -0.00271 \\ \mathrm{H} & 3.63465 & -0.63333 & -0.88345 \\ \mathrm{H} & 3.63344 & -0.63798 & 0.88642 \\ \mathrm{H} & -0.41822 & 1.96586 & -0.00011 \\ \mathrm{H} & -4.32592 & 0.26874 & 0.00004 \\ \mathrm{H} & -3.39045 & -0.97263 & -0.89555 \\ \mathrm{H} & -3.39042 & -0.97275 & 0.89545\end{array}$


Table S2. Structure, energy and Cartesian coordinate of $\mathbf{2 b}$

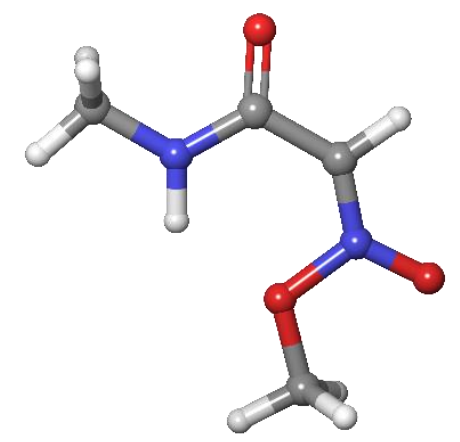

$\mathbf{2 b}$ (no imaginary frequencies)

B3LYP/6-311+G(d,p)-IEFPCM $\left(\mathrm{CHCl}_{3}\right) / / \mathrm{B} 3 \mathrm{LYP} / 6-31 \mathrm{G}+(\mathrm{d}, \mathrm{p})$ :

Gibbs Free Energy (a.u.) $\quad=-492.370807$

B3LYP/6-311+G(d,p)-IEFPCM $\left(\mathrm{CHCl}_{3}\right)$ :

Electronic energy (a.u.) $=-492.466086$

B3LYP/6-31+G(d,p):

$$
\text { Zero-point correction (a.u.) }=0.132561
$$

Thermal correction to Energy (a.u.) $=0.142960$

Thermal correction to Enthalpy (a.u.) $=0.143905$

Thermal correction to Gibbs Free Energy (a.u.) $=0.095279$

$\begin{array}{lrrr}\mathrm{N} & 1.46920 & 0.73642 & -0.00002 \\ \mathrm{C} & 1.28204 & -0.60867 & -0.00000 \\ \mathrm{O} & 2.22472 & -1.40770 & 0.00001 \\ \mathrm{C} & 2.81364 & 1.29312 & 0.00001 \\ \mathrm{C} & -0.08510 & -1.18889 & -0.00000 \\ \mathrm{~N} & -1.26838 & -0.62010 & -0.00001 \\ \mathrm{O} & -1.25821 & 0.81985 & -0.00003 \\ \mathrm{C} & -2.58557 & 1.37676 & 0.00002 \\ \mathrm{O} & -2.37881 & -1.16954 & 0.00000 \\ \mathrm{H} & 0.66350 & 1.34229 & 0.00000 \\ \mathrm{H} & 3.37084 & 0.96927 & -0.88462 \\ \mathrm{H} & 2.73748 & 2.38204 & -0.00015 \\ \mathrm{H} & 3.37073 & 0.96952 & 0.88480 \\ \mathrm{H} & -0.12992 & -2.26886 & 0.00001 \\ \mathrm{H} & -3.13613 & 1.08261 & -0.89514 \\ \mathrm{H} & -3.13606 & 1.08262 & 0.89522 \\ \mathrm{H} & -2.39781 & 2.45146 & 0.00000\end{array}$


Table S3. Crystallographic data of $\mathbf{1 1}$

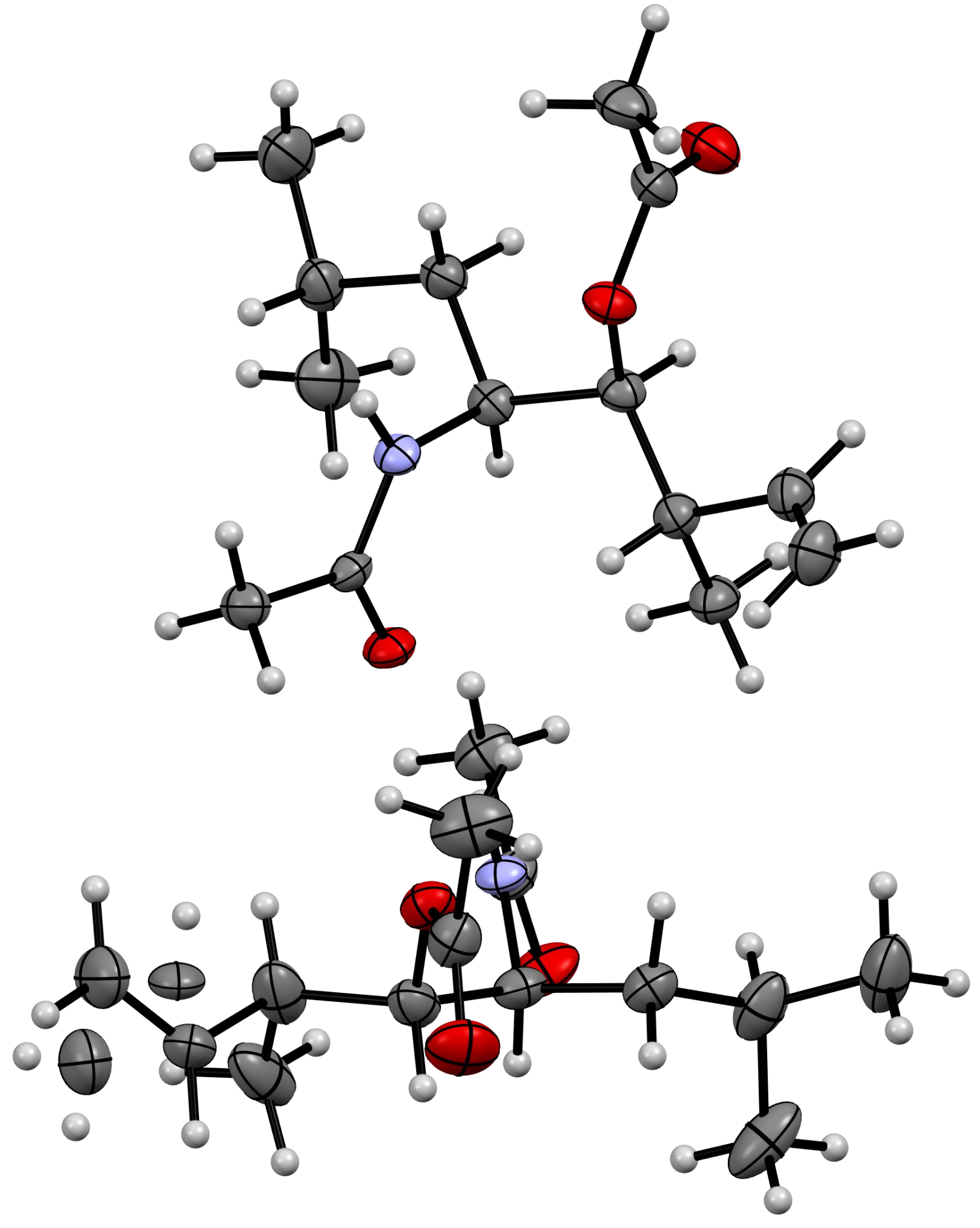

X-ray crystallographic structure of $\mathbf{1 1}$ with thermal ellipsoids at the $50 \%$ probability level 


\begin{tabular}{|c|c|c|}
\hline CCDC number & \multicolumn{2}{|l|}{2058723} \\
\hline Chemical formula & \multicolumn{2}{|l|}{$\mathrm{C}_{14} \mathrm{H}_{25} \mathrm{NO}_{3}$} \\
\hline Formula weight & \multicolumn{2}{|l|}{$255.35 \mathrm{~g} / \mathrm{mol}$} \\
\hline Temperature & \multicolumn{2}{|l|}{$100(2) \mathrm{K}$} \\
\hline Wavelength & \multicolumn{2}{|l|}{$1.54178 \AA$} \\
\hline Crystal size & \multicolumn{2}{|c|}{$0.233 \times 0.619 \times 1.322 \mathrm{~mm}$} \\
\hline Crystal habit & \multicolumn{2}{|c|}{ clear light colourless prism } \\
\hline Crystal system & \multicolumn{2}{|l|}{ monoclinic } \\
\hline Space group & \multicolumn{2}{|l|}{ P 1211} \\
\hline \multirow[t]{3}{*}{ Unit cell dimensions } & $\mathrm{a}=9.0402(3) \AA$ & $\alpha=90^{\circ}$ \\
\hline & $\mathrm{b}=18.8773(6) \AA$ & $\beta=102.346(2)^{\circ}$ \\
\hline & $c=9.5047(3) \AA$ & $\gamma=90^{\circ}$ \\
\hline Volume & \multicolumn{2}{|l|}{$1584.51(9) \AA^{3}$} \\
\hline $\mathbf{Z}$ & \multicolumn{2}{|l|}{4} \\
\hline Density (calculated) & \multicolumn{2}{|l|}{$1.070 \mathrm{~g} / \mathrm{cm}^{3}$} \\
\hline Absorption coefficient & \multicolumn{2}{|l|}{$0.596 \mathrm{~mm}^{-1}$} \\
\hline $\mathbf{F}(000)$ & \multicolumn{2}{|l|}{560} \\
\hline Diffractometer & \multicolumn{2}{|c|}{ Bruker D8 goniometer } \\
\hline Theta range for data collection & \multicolumn{2}{|l|}{4.68 to $62.45^{\circ}$} \\
\hline Index ranges & \multicolumn{2}{|c|}{$-10<=\mathrm{h}<=10,-21<=\mathrm{k}<=21,-10<=1<=10$} \\
\hline Reflections collected & \multicolumn{2}{|l|}{18828} \\
\hline Independent reflections & \multicolumn{2}{|c|}{$5013[\mathrm{R}(\mathrm{int})=0.0417]$} \\
\hline Coverage of independent reflections & \multicolumn{2}{|l|}{$99.4 \%$} \\
\hline Absorption correction & \multicolumn{2}{|l|}{ Multi-Scan } \\
\hline Max. and min. transmission & \multicolumn{2}{|l|}{0.8740 and 0.5060} \\
\hline Structure solution technique & \multicolumn{2}{|l|}{ direct methods } \\
\hline Structure solution program & \multicolumn{2}{|c|}{ SHELXT 2014/5 (Sheldrick, 2014) } \\
\hline Refinement method & \multicolumn{2}{|c|}{ Full-matrix least-squares on F2 } \\
\hline Refinement program & \multicolumn{2}{|c|}{ SHELXL-2018/3 (Sheldrick, 2018) } \\
\hline Function minimized & \multicolumn{2}{|l|}{$\Sigma \mathrm{w}\left(\mathrm{F}_{\mathrm{o}}^{2}-\mathrm{F}_{\mathrm{c}}^{2}\right)^{2}$} \\
\hline Data / restraints / parameters & \multicolumn{2}{|l|}{$5013 / 1 / 362$} \\
\hline Goodness-of-fit on F2 & 0.870 & \\
\hline Final $R$ indices & 4879 data; $\mathrm{I}>2 \sigma(\mathrm{I})$ & $\mathrm{R} 1=0.0334, \mathrm{wR} 2=0.0895$ \\
\hline & all data & $\mathrm{R} 1=0.0345, \mathrm{wR} 2=0.0913$ \\
\hline Weighting scheme & $\mathrm{w}=1 /\left[\sigma^{2}\left(\mathrm{~F}_{\mathrm{o}}^{2}\right)+(0.0\right.$ & )$\left.^{2}+0.3384 \mathrm{P}\right]$ where $\mathrm{P}=\left(\mathrm{F}_{\mathrm{o}}^{2}+2 \mathrm{~F}_{\mathrm{c}}^{2}\right) / 3$ \\
\hline Absolute structure parameter & $-0.06(7)$ & \\
\hline Largest diff. peak and hole & 0.117 and $-0.151 \mathrm{e}$ & \\
\hline R.M.S. deviation from mean & $0.034 \mathrm{e}^{-3}$ & \\
\hline
\end{tabular}

